\title{
Subgame perfect equilibria in majoritarian bargaining
}

Citation for published version (APA):

Herings, P. J. J., Meshalkin, A. V., \& Predtetchinski, A. (2013). Subgame perfect equilibria in majoritarian bargaining. Maastricht University, Graduate School of Business and Economics. GSBE Research Memoranda No. 072 https://doi.org/10.26481/umagsb.2013072

Document status and date:

Published: 01/01/2013

DOI:

10.26481/umagsb.2013072

Document Version:

Publisher's PDF, also known as Version of record

\section{Please check the document version of this publication:}

- A submitted manuscript is the version of the article upon submission and before peer-review. There can be important differences between the submitted version and the official published version of record.

People interested in the research are advised to contact the author for the final version of the publication, or visit the DOI to the publisher's website.

- The final author version and the galley proof are versions of the publication after peer review.

- The final published version features the final layout of the paper including the volume, issue and page numbers.

Link to publication

\footnotetext{
General rights rights.

- You may freely distribute the URL identifying the publication in the public portal. please follow below link for the End User Agreement:

www.umlib.nl/taverne-license

Take down policy

If you believe that this document breaches copyright please contact us at:

repository@maastrichtuniversity.nl

providing details and we will investigate your claim.
}

Copyright and moral rights for the publications made accessible in the public portal are retained by the authors and/or other copyright owners and it is a condition of accessing publications that users recognise and abide by the legal requirements associated with these

- Users may download and print one copy of any publication from the public portal for the purpose of private study or research.

- You may not further distribute the material or use it for any profit-making activity or commercial gain

If the publication is distributed under the terms of Article $25 \mathrm{fa}$ of the Dutch Copyright Act, indicated by the "Taverne" license above, 


\section{Maastricht University}

P. Jean-Jacques Herings,

Andrey Meshalkin,

Arkadi Predtetchinski

Subgame Perfect Equilibria in Majoritarian Bargaining

RM/13/072

\section{GSBE}

Maastricht University School of Business and Economics

Graduate School of Business and Economics

P.O Box 616

NL- 6200 MD Maastricht

The Netherlands 


\title{
Subgame Perfect Equilibria in Majoritarian Bargaining
}

\author{
P. Jean-Jacques Herings*, Andrey Meshalkin†, Arkadi Predtetchinski ${ }^{\ddagger}$
}

December 9, 2013

\begin{abstract}
We study the division of a surplus under majoritarian bargaining in the threeperson case. In a stationary equilibrium as derived by Baron and Ferejohn (1989), the proposer offers one third times the discount factor of the surplus to a second player and allocates no payoff to the third player, a proposal which is accepted without delay. Laboratory experiments show various deviations from this equilibrium, where different offers are typically made and delay may occur before acceptance. We address the issue to what extent these findings are compatible with subgame perfect equilibrium and characterize the set of subgame perfect equilibrium payoffs for any value of the discount factor. We show that for any proposal in the interior of the space of possible agreements there exists a discount factor such that the proposal is made and accepted. We characterize the values of the discount factor for which equilibria with one-period delay exist. We show that any amount of equilibrium delay is possible and we construct subgame perfect equilibria such that arbitrary long delay occurs with probability one.
\end{abstract}

KEYwords: Dynamic games, bargaining, majoritarian voting, subgame perfect equilibrium, delay of acceptance.

JEL CODES: C72, C78.

*P.J.J. Herings (P.Herings@maastrichtuniversity.nl). Department of Economics, Maastricht University. $\dagger$ A. Meshalkin (A.Rybakov@maastrichtuniversity.nl), Department of Economics, Maastricht University.

${ }^{\ddagger}$ A. Predtetchinski (A.Predtetchinski@maastrichtuniversity.nl), Department of Economics, Maastricht University. 


\section{Introduction}

Many decisions in legislatures involve the task of allocating resources among constituencies with diverse and often opposing preferences. To analyze such decisions, many authors have studied extensions of the Rubinstein (1982) bargaining model to collective choice problems, see for instance Baron and Ferejohn (1989), Harrington (1990), Baron and Kalai (1993), and Banks and Duggan (2000). These papers study multilateral bargaining games, where an alternative is accepted if it is approved by a set of players that belongs to a collection of decisive coalitions. This approach makes it possible to study a variety of important institutional set-ups, including the one of majority voting.

The Baron and Ferejohn (1989) model, hereafter BF model, is the most frequently used model to study legislative bargaining. Baron and Ferejohn (1989) consider a bargaining game with infinite time horizon, where the proposer selection process is modeled by timeinvariant recognition probabilities, and acceptance by a simple majority is sufficient to implement a proposal. Eraslan (2002) shows that stationary subgame perfect equilibrium payoffs are unique for a rather general specification of the BF model, and Eraslan and McLennan (2013) show uniqueness of such equilibrium payoffs in an even more general model. In any stationary equilibrium of the BF model, the proposer offers $\delta / n$ to half of the responding players, nothing to the other responders, and keeps the remainder. Such a proposal is accepted without delay. The equilibrium therefore predicts substantial proposer power, the formation of a minimum winning coalition, and absence of delay in reaching an agreement.

The BF result has been used as the theoretical benchmark in many papers in the experimental literature. McKelvey (1991) studies a bargaining game with three voters and a discrete policy space consisting of three alternatives. Fréchette, Kagel, and Lehrer (2003) use the BF model with five players as their main treatment and study the effects of open versus closed amendment rules. Fréchette, Kagel, and Morelli (2005) use the BF specification with three players and a discount factor of $1 / 2$ in their treatments, as well as the case without discounting, and focus on the influence of nominal bargaining power on bargaining outcomes. Diermeier and Morton (2006) consider a version of the BF model with a finite time horizon and heterogeneous recognition probabilities. Breitmoser and Tan (2010) consider the BF specification with three players in their treatments and study in particular whether fairness can explain some of the experimental findings. Kagel, Sung, and Winter (2010) are interested in the consequences of veto power in committees and use the BF specification with three players and a discount factor of 0.95 as a benchmark. Miller and Vanberg (2013) use the BF model with three players and a discount factor of 0.9 when comparing the costs of reaching agreement under majority and unanimity rule. 
The experimental literature finds that the predictions of the stationary subgame perfect equilibrium in the BF model are violated in several ways, although certain qualitative features like the presence of proposer power and a tendency to form minimum winning coalitions are found. The most striking feature of the data is that proposers make overly generous offers to the responding players and keep a much smaller share for themselves than predicted by the stationary equilibrium. Minimum winning coalitions are not always formed and even an equal sharing of the surplus with all players occurs with significantly positive probability. Contrary to the BF prediction of immediate acceptance, there is a significant number of observations with delay before a proposal is accepted, in particular when the discount factor is sufficiently high.

McKelvey (1991) conjectures that subjects may offer too much to potential coalition partners because of fear of retaliation in later rounds and Diermeier and Morton (2006) report the use of non-stationary strategies. For instance, subjects who vote to reject a proposal on average receive a higher payoff from the new proposal, which is a violation of stationarity since under stationarity irrelevant parts of the history should be of no influence to future behavior. Many authors, most notably Rubinstein (1991), have argued against the use of stationary strategies in bargaining. We are therefore interested in the question whether proposals like for instance equal sharing of the surplus are compatible with subgame perfect equilibrium and whether delay is possible in subgame perfect equilibria when stationarity is not imposed.

In this paper we study the BF model with three players and we characterize the set of subgame perfect equilibrium utilities for any value of the discount factor. When the discount factor goes to zero, the set of subgame equilibrium payoffs shrinks to the unit vector and the entire surplus is allocated to the player who is recognized as the proposer in period zero. When the discount factor goes to one, the set of subgame equilibria payoffs expands to the set of feasible payoffs. In particular, all inefficient outcomes are supported as subgame perfect equilibria when the discount factor is sufficiently high. Our characterization of the set of subgame perfect equilibrium utilities is entirely constructive.

For any vector in the interior of the set of feasible payoffs there exist a discount factor such that this vector is proposed and accepted in period 0 in a subgame perfect equilibrium in pure strategies. Nevertheless, the usual statement of the folk theorem, stating that for a sufficiently high discount factor any individually rational payoff is a subgame perfect equilibrium payoff, does not hold in the BF model with three players.

Baron and Ferejohn (1989) prove the folk theorem for a game with at least five players. It follows from our characterization of the set of subgame perfect equilibrium utilities that this result does not extend to the case with three players. Chatterjee, Dutta, Ray, and Sengupta (1993) consider bargaining in the context of strictly superadditive transferable 
utility games under the rejector-proposes protocol and show the usual statement of the folk theorem that for a sufficiently high discount factor any individually rational outcome is possible. Note that the BF model violates strict superadditivity when viewed as a transferable utility game. Norman (2002) studies a finite horizon version of the BF model and shows for the case with at least five players that any interior division of the surplus can be supported as a subgame perfect equilibrium outcome if players are sufficiently patient and there are sufficiently many rounds of bargaining. He also shows indeterminacy of the equilibrium outcome whenever there are at least three players and at least three bargaining rounds.

The BF model with three players is a borderline case where the usual statement of the folk theorem does not apply, but the set of subgame perfect equilibria can be sizable. Recall that in the Rubinstein (1982) model with two players, there is a unique subgame perfect equilibrium. Cho and Duggan (2009) analyze a version of the BF model where an arbitrary number of players bargains on a one-dimensional ideological spectrum of policies and agents have single-peaked preferences over policies. Under sequential voting and some mild assumptions on the order of voting, they find that only the median ideal alternative can be supported in a subgame perfect equilibrium. Cho and Duggan (2013) demonstrate that this result does not carry over to simultaneous and publicly observed voting, even when voting is assumed to be stage-undominated and even obtain the support of arbitrary outcomes for arbitrary positive discount factors.

For the BF model with three players, we provide necessary and sufficient conditions for one period of delay before acceptance takes place. We characterize the threshold such that, for any value of the discount factor greater than the threshold, one period of delay is possible and for any value of the discount factor smaller than the threshold all subgame perfect equilibria are characterized by immediate acceptance. We also demonstrate the possibility of arbitrary long delay before acceptance takes place in a subgame perfect equilibrium when the discount factor is sufficiently high.

For low values of the discount factors, the offers as observed in experiments are not consistent with stationary equilibrium, and are not even consistent with subgame perfect equilibrium. For instance, when the discount factor is equal to $1 / 2$, Fréchette, Kagel, and Morelli (2005) find an average share of the proposer for accepted offers equal to 0.50, lower than the lowest possible share of 0.78 in a subgame perfect equilibrium, which does not differ much from the stationary equilibrium share of 0.83 . For higher values of the discount factor, the literature reports on experiments with discount factors equal to 0.9 , 0.95, and 1, the experimental findings on accepted offers are not in contradiction with the predictions of subgame perfect equilibrium. Regarding delay before reaching an agreement, the experimental literature find little delay for experienced subjects when the discount 
factor is equal to 0.5 , but substantial delay when the discount factor is equal to 0.9 or higher. We find that the critical discount factor needed to have subgame perfect equilibria with one-period delay is slightly above 0.8 .

The rest of the paper is organized as follows. In Section 2 we describe the BF model and derive bounds on subgame perfect equilibrium utilities as a function of the discount factor. In Section 3 we characterize the subgame perfect equilibrium utilities conditional on the selection of a proposer and argue that the bounds in Section 2 are tight. The explicit construction of the subgame perfect equilibria that reach the bounds is relegated to the appendix. In Section 4 we provide necessary and sufficient conditions on the discount factor for one period of delay before acceptance takes place. In Section 5 we investigate the possibility of arbitrary long delay before acceptance takes place in a subgame perfect equilibrium. Section 6 concludes.

\section{The bounds for subgame perfect equilibrium payoffs}

We consider the three player version of the BF model. The set of players $N=\{1,2,3\}$ has to agree on the choice of a payoff vector in the set of feasible payoffs $V=\left\{x \in \mathbb{R}_{+}^{3}\right.$, $\left.x_{1}+x_{2}+x_{3} \leq 1\right\}$. In each time period $t=0,1,2, \ldots$ nature selects a proposer and the order of the responders by means of time-invariant recognition probabilities. More precisely, in the beginning of period $t=0,1,2, \ldots$ nature selects each permutation $\pi^{t}:\{1,2,3\} \rightarrow N$ in the set of six possible permutations $\Pi$ with equal probability. The choice of the permutation is independent across time periods. Player $\pi^{t}(1)$ makes a proposal $x^{t} \in V$. Next Player $\pi^{t}(2)$ responds by accepting or rejecting the proposal. If $\pi^{t}(2)$ accepts the proposal, the game ends, and the proposal $x^{t}$ is implemented. Otherwise, Player $\pi^{t}(3)$ reacts to the proposal. If Player $\pi^{t}(3)$ accepts, the game ends. If Player $\pi^{t}(3)$ rejects the proposal, period $t+1$ begins. The utility of player $i \in N$ who receives outcome $x_{i}$ in period $t$ is $\delta^{t} x_{i}$, where $\delta \in[0,1)$ is the common discount factor. If no agreement is ever reached, payoffs are 0 .

A history $h$ is the sequence of all actions that have occurred before a particular decision node in the game. For simplicity we suppress the elements of the sequence pertaining to the moves by the responding players: it is understood that in any non-terminal history both responders have rejected all proposals to date. With this convention, for $t=0,1, \ldots$ any non-terminal history is of one of the following two types:

1. $h \in H_{1}^{t}$ if and only if $h$ is of the form $\left(\pi^{0}, x^{0}, \ldots, \pi^{t-1}, x^{t-1}, \pi^{t}\right)$,

2. $h \in H_{2}^{t}$ if and only if $h$ is of the form $\left(\pi^{0}, x^{0}, \ldots, \pi^{t-1}, x^{t-1}, \pi^{t}, x^{t}\right)$, 
where for every $k \in\{0, \ldots, t\}, \pi^{k}$ is a permutation of $N$ and $x^{k} \in V$. After a history $h \in H_{1}^{t}$, the proposer makes a move and, after a history $h \in H_{2}^{t}$, a responder accepts or rejects the current proposal. Histories in $H_{1}^{t}$ are therefore called proposer histories and those in $H_{2}^{t}$ responder histories.

A pure strategy for Player $i \in N$ assigns to every history $h=\left(\pi^{0}, x^{0}, \ldots, \pi^{t}\right) \in H_{1}^{t}$ with $\pi^{t}(1)=i$ an element of $V$, and to every history $h \in H_{2}^{t}$ with $\pi^{t}(1) \neq i$ an accept/reject decision. All our results make only use of pure strategy profiles. It is an easy exercise to verify that the bounds we present on equilibrium utilities carry over to mixed strategy profiles as well.

A strategy is stationary if it does not depend on payoff irrelevant parts of the history, see Maskin and Tirole (2001) for a precise formulation of the notion of stationarity. For the BF model, stationarity means that a player makes the same proposal at every history in $H_{1}^{t}$ where he is recognized as a proposer and bases his accept/reject decisions only on the current proposal and the identity of the player voting next to him in the current voting round.

The following result follows from the analysis in Baron and Ferejohn (1989).

Theorem 2.1 For every $\delta \in[0,1)$, a strategy profile is a subgame perfect equilibrium in stationary strategies if and only if it has the following form: (1) the recognized player offers $\delta / 3$ to one responding player and keeps $1-\delta / 3$ for himself; (2) a responding player accepts any proposal in which he receives at least $\delta / 3$ and rejects otherwise; (3) all players receive $\delta / 3$ as responding player with ex ante probability equal to $1 / 3$.

In equilibrium, the recognized player forms a minimum winning coalition with one responding player, offers the responding player exactly his continuation utility, offers nothing to the third player, and keeps the remainder of the surplus. The responding player who is part of the minimum winning coalition accepts this proposal. ${ }^{1}$ The equilibrium in Theorem 2.1 is not unique, since there is some indeterminacy in the selection by the proposer of the player who joins the minimum winning coalition. However, all strategy profiles described in Theorem 2.1 lead to the same equilibrium payoffs. Baron and Ferejohn (1989) show that this result extends to the case with an odd number $n$ of players, where now $\delta(n-1) / 2 n$ is offered to $(n-1) / 2$ players, 0 to the other players, and the remainder is kept by the proposer. It follows from Eraslan (2002) that stationary subgame perfect equilibrium payoffs are unique, also when mixed strategies are allowed for.

We now drop the stationarity assumption on strategies. The set of all subgame perfect equilibrium (SPE) payoff vectors is denoted by $U$, a subset of $V$. For $i \in N$, let $\bar{u}_{i}$ be the

\footnotetext{
${ }^{1}$ On top of stationarity, it is assumed in Baron and Ferejohn (1989) that whenever indifferent, a responder accepts a proposal.
} 


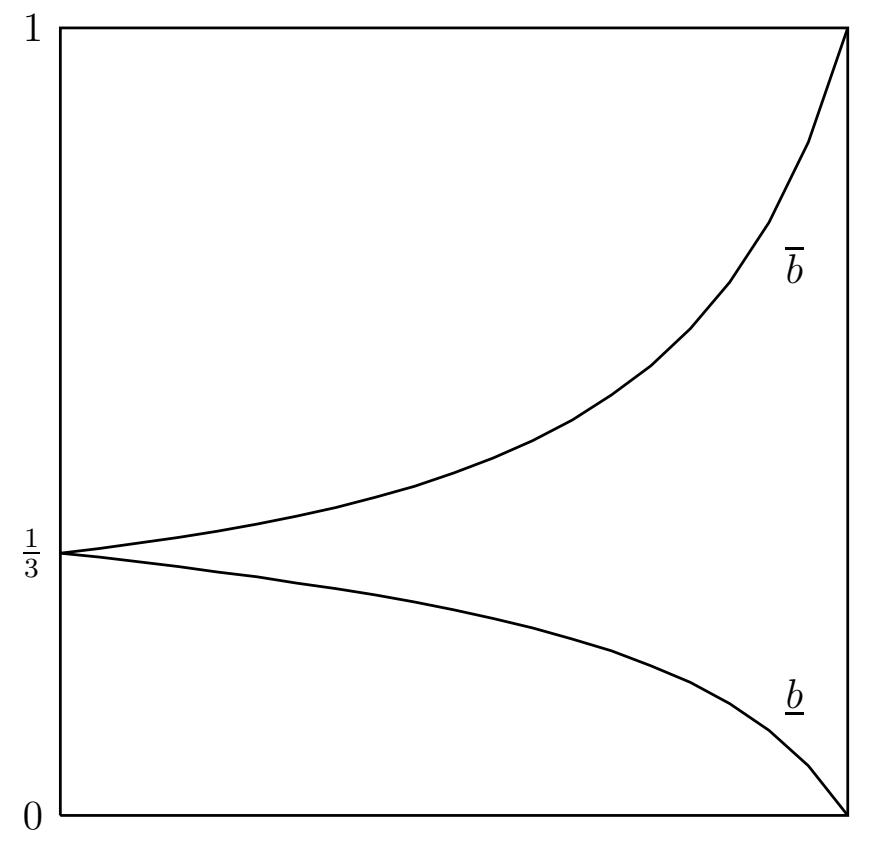

Figure 1: $\underline{b}$ and $\bar{b}$ as functions of $\delta$.

supremum of SPE payoffs for Player $i$ and $\underline{u}_{i}$ be the infimum, so

$$
\begin{aligned}
& \bar{u}_{i}=\sup \left\{u_{i} \in \mathbb{R} \mid u \in U\right\}, \\
& \underline{u}_{i}=\inf \left\{u_{i} \in \mathbb{R} \mid u \in U\right\} .
\end{aligned}
$$

We define the real numbers $\underline{b}$ and $\bar{b}$ by

$$
\begin{aligned}
& \underline{b}=\frac{3-3 \delta}{9-6 \delta-\delta^{2}}, \\
& \bar{b}=\frac{3-\delta}{9-6 \delta-\delta^{2}} .
\end{aligned}
$$

Figure 1 plots $\underline{b}$ and $\bar{b}$ as a function of $\delta$. The following result characterizes the subgame perfect equilibrium utilities as a function of the discount factor.

Theorem 2.2 It holds that $\bar{u}_{1}=\bar{u}_{2}=\bar{u}_{3}=\bar{b}$ and $\underline{u}_{1}=\underline{u}_{2}=\underline{u}_{3}=\underline{b}$.

The proof of Theorem 2.2 consists of two parts. The first part, carried out in the remainder of this section, consists of showing that in any SPE the payoff to any player is bounded from below by $\underline{b}$ and from above by $\bar{b}$, that is $\underline{b} \leq \underline{u}_{i}$ and $\bar{u}_{i} \leq \bar{b}$. In the next section we show that these bounds are tight: we explicitly construct an SPE where one player receives a payoff of $\bar{b}$ and another player a payoff of $\underline{b}$.

Due to the symmetry of the game it is clear that $\bar{u}_{1}=\bar{u}_{2}=\bar{u}_{3}$ and $\underline{u}_{1}=\underline{u}_{2}=\underline{u}_{3}$. We henceforth write simply $\bar{u}$ instead of $\bar{u}_{i}$ and $\underline{u}$ instead of $\underline{u}_{i}$.

The first claim puts an upperbound of 1 on the sum of $\underline{u}$ and $\bar{u}$. 
Claim 2.3 It holds that $\underline{u}+\bar{u} \leq 1$.

Proof: Consider some $u \in U$. Since $u \in V$, it holds that $u_{1}+u_{2} \leq u_{1}+u_{2}+u_{3} \leq 1$, and therefore $u_{1} \leq 1-u_{2}$. We have that

$$
\bar{u}=\sup \left\{u_{1} \mid u \in U\right\} \leq \sup \left\{1-u_{2} \mid u \in U\right\}=1-\inf \left\{u_{2} \mid u \in U\right\}=1-\underline{u} .
$$

Our next claim puts bounds on the behavior of responders in an SPE.

Claim 2.4 Consider the subgame following the period 0 proposer history $h=(\pi)$. In any SPE $s$ it holds that

[1] A proposal $x \in V$ by Player $\pi(1)$ such that $x_{\pi(2)}>\delta \bar{u}$ or $x_{\pi(3)}>\delta \bar{u}$ is accepted by Player $\pi(2)$ or by Player $\pi(3)$.

[2] A proposal $x \in V$ by Player $\pi(1)$ such that $x_{\pi(2)}<\delta \underline{u}$ and $x_{\pi(3)}<\delta \underline{u}$ is rejected by both Players $\pi(2)$ and $\pi(3)$.

Proof: [1] Let $x \in V$ be such that $x_{i}>\delta \bar{u}$ for some $i \in\{\pi(2), \pi(3)\}$. Suppose that according to the strategy profile $s$ the proposal $x$ is rejected both by Player $\pi(2)$ and by Player $\pi(3)$. Consider the responder history $(h, x)$. The payoff to Player $i$ of the strategy profile $s$ at $(h, x)$ is at most $\delta \bar{u}$. On the other hand, accepting the proposal $x$ yields Player $i$ a payoff of $x_{i}$. Thus Player $i$ has a profitable deviation at $(h, x)$, leading to a contradiction.

[2] Let $x \in V$ be such that $x_{i}<\delta \underline{u}$ for both $i \in\{\pi(2), \pi(3)\}$. Consider Player $\pi(3)$ 's action at the responder history $(h, x)$. Since accepting $x$ yields a payoff of $x_{\pi(3)}$ and rejecting $x$ gives a payoff of at least $\delta \underline{u}$, Player $\pi(3)$ must reject. Consider now Player $\pi(2)$ 's action at $(h, x)$. A rejection of $x$ by Player $\pi(2)$ is followed by the rejection of $x$ by Player $\pi(3)$ and so yields Player $\pi(2)$ a payoff of at least $\delta \underline{u}$, whereas acceptance leads to a payoff of only $x_{\pi(2)}$. We conclude that Player $\pi(2)$ must reject $x$.

The statement in [1] of Claim 2.4 is subtle. It considers a proposal where one or both responding players receive more than $\delta \bar{u}$. The claim is that such a proposal is accepted, but the statement does not specify by whom. Indeed, if both responding players receive more than $\delta \bar{u}$, then Player $\pi(2)$ can safely reject such a proposal in the knowledge that Player $\pi(3)$ is going to accept it. Similarly, if the proposal is such that only Player $\pi(3)$ receives more than $\delta \bar{u}$, it could still be that Player $\pi(2)$ accepts it, since Player $\pi(2)$ is indifferent whether such a proposal is accepted by himself or by Player $\pi(3)$.

The following claim derives bounds on SPE utilities conditional on the selection of the proposer. 
Claim 2.5 Consider the subgame following the period 0 proposer history $h=(\pi)$ and let $v=\left(v_{1}, v_{2}, v_{3}\right)$ be the payoffs of an SPE s conditional on the subgame being reached. It holds that

$$
\begin{aligned}
& {[1] \quad v_{\pi(1)} \geq 1-\delta \bar{u}} \\
& {[2] \quad v_{\pi(1)} \leq 1-\delta \underline{u},} \\
& {[3] \quad v_{\pi(2)} \leq \delta \bar{u} \text { and } v_{\pi(3)} \leq \delta \bar{u}}
\end{aligned}
$$

Proof: [1] By Claim 2.4 the proposal $x$ where $x_{\pi(1)}=1-\delta \bar{u}-\epsilon, x_{\pi(2)}=\delta \bar{u}+\epsilon$, and $x_{\pi(3)}=0$ is accepted for each $\epsilon>0$. Since $s$ is an SPE we have $v_{\pi(1)} \geq 1-\delta \bar{u}-\epsilon$ for each $\epsilon>0$. It follows that $v_{\pi(1)} \geq 1-\delta \bar{u}$.

[2] Let $x$ be Player $\pi(1)$ 's proposal at $h$ under the strategy profile $s$. Suppose first that $x$ is rejected under $s$. Since this results in at least one period of delay, it follows that $v_{\pi(1)} \leq \delta \bar{u}$. It follows from Claim 2.3 that $\delta \bar{u} \leq 1-\delta \underline{u}$ and therefore that $v_{\pi(1)} \leq 1-\delta \underline{u}$. Suppose now $x$ is accepted. Then by Claim 2.4 it is the case that $x_{\pi(2)} \geq \delta \underline{u}$ or $x_{\pi(3)} \geq \delta \underline{u}$. Therefore $v_{\pi(1)}=x_{\pi(1)} \leq 1-x_{\pi(2)}-x_{\pi(3)} \leq 1-\delta \underline{u}$.

[3] We have $v_{\pi(2)} \leq 1-v_{\pi(1)}-v_{\pi(3)} \leq 1-v_{\pi(1)} \leq \delta \bar{u}$, where the last inequality follows from [1]. The argument for $v_{\pi(3)}$ is similar.

The equilibrium utility conditional on being the initial proposer is in between $1-\delta \bar{u}$ and $1-\delta \underline{u}$. Responders have equilibrium utilities bounded from above by $\delta \bar{u}$. The result carries over to subgames in later periods $t$, except that discounting implies that all utilities should be multiplied by $\delta^{t}$.

We now use the claims derived so far to derive a lower bound on $\underline{u}$ in terms of $\delta$ and $\bar{u}$, and an upper bound on $\bar{u}$ in terms of $\delta$ and $\underline{u}$.

Claim 2.6 It holds that

$$
\frac{1-\delta \bar{u}}{3} \leq \underline{u} \leq \bar{u} \leq \frac{1-\delta \underline{u}}{3-2 \delta}
$$

Proof: Let $s$ be an SPE with payoffs $u \in U$. For each permutation $\pi \in \Pi$, let $v^{\pi}=$ $\left(v_{1}^{\pi}, v_{2}^{\pi}, v_{3}^{\pi}\right)$ be the payoff of $s$ conditional on nature choosing $\pi$ as the initial permutation. Since each permutation is equally likely, we have

$$
u=\frac{1}{6} \sum_{\pi \in \Pi} v^{\pi}
$$

For a permutation $\pi \in\{(1,2,3),(1,3,2)\}$ it holds that Player 1 is the proposer. By parts [1] and [2] of Claim 2.5 it holds that $1-\delta \bar{u} \leq v_{1}^{\pi} \leq 1-\delta \underline{u}$. 
Next, consider a permutation $\pi \in \Pi \backslash\{(1,2,3),(1,3,2)\}$. Now Player 1 is a responding player and so $0 \leq v_{1}^{\pi} \leq \delta \bar{u}$ by part [3] of Claim 2.5. We conclude that

$$
\frac{2}{6}(1-\delta \bar{u})+\frac{4}{6} 0 \leq u_{1} \leq \frac{2}{6}(1-\delta \underline{u})+\frac{4}{6} \delta \bar{u} .
$$

Since the inequalities hold for every $u \in U$, we have

$$
\frac{1}{3}(1-\delta \bar{u}) \leq \underline{u} \leq \bar{u} \leq \frac{1}{3}(1-\delta \underline{u})+\frac{2}{3} \delta \bar{u} .
$$

Rearranging the last inequality yields the result.

We are now in a position to prove the first half of Theorem 2.2. The payoff to any player in any SPE of the game is bounded from below by $\underline{b}$ and from above by $\bar{b}$.

Theorem 2.7 It holds that $\underline{b} \leq \underline{u} \leq \bar{u} \leq \bar{b}$.

Proof: Define the functions $f, g:[0,1] \rightarrow[0,1]$ by

$$
f(y)=\frac{1-\delta y}{3} \text { and } g(y)=\frac{1-\delta y}{3-2 \delta}
$$

and let $h=g \circ f$. Then the function $h$ is defined by

$$
h(y)=\frac{1-\delta\left(\frac{1-\delta y}{3}\right)}{3-2 \delta}=\frac{3-\delta+\delta^{2} y}{9-6 \delta}, \quad y \in[0,1] .
$$

By Claim 2.6, we have that

$$
f(\bar{u}) \leq \underline{u} \leq \bar{u} \leq g(\underline{u})
$$

Using the fact that $g$ is a non-increasing function, we obtain $\bar{u} \leq g(\underline{u}) \leq g(f(\bar{u}))$, and hence $\bar{u} \leq h(\bar{u})$. Since $h$ is a non-decreasing function, we can iterate the last inequality to obtain $\bar{u} \leq h^{n}(\bar{u})$.

It is easy to see that $h$ is a contraction and that $\bar{b}$ is a fixed point of $h$. By the Banach contraction theorem it follows that $h^{n}(\bar{u})$ converges to $\bar{b}$, so we have shown that $\bar{u} \leq \bar{b}$.

An easy computation shows that $\underline{b}=f(\bar{b})$. Since $f$ is a non-increasing function, we have that

$$
\underline{b}=f(\bar{b}) \leq f(\bar{u}) \leq \underline{u}
$$

which completes the proof. 


\section{Explicit subgame perfect equilibria}

In the previous section we have shown that SPE utilities are bounded from below by $\underline{b}$ and from above by $\bar{b}$. In this section we show that these bounds are tight by constructing an SPE yielding Player 2 a payoff of $\bar{b}$ and Player 3 a payoff of $\underline{b}$.

We define

$$
\begin{aligned}
& V_{1}=\left\{v \in V \mid v_{1} \geq 1-\delta \bar{b} \text { and }\left[v_{2} \geq \delta \underline{b} \text { or } v_{3} \geq \delta \underline{b}\right]\right\}, \\
& V_{2}=\left\{v \in V \mid v_{2} \geq 1-\delta \bar{b} \text { and }\left[v_{3} \geq \delta \underline{b} \text { or } v_{1} \geq \delta \underline{b}\right]\right\}, \\
& V_{3}=\left\{v \in V \mid v_{3} \geq 1-\delta \bar{b} \text { and }\left[v_{1} \geq \delta \underline{b} \text { or } v_{2} \geq \delta \underline{b}\right]\right\} .
\end{aligned}
$$

For $i \in N$, the set $V_{i}$ gives the payoffs that can be obtained as SPE utilities conditional on reaching a subgame in Period 0 where Player $i$ is selected as a proposer.

Theorem 3.1 Choose $a_{1} \in V_{1}, a_{2} \in V_{2}$, and $a_{3} \in V_{3}$. Then there exists an SPE $s$ such that for each permutation $\pi^{0}$ the proposal $a_{\pi^{0}(1)}$ is made and accepted in period 0.

The constructive proof of Theorem 3.1 can be found in the appendix. Using Theorem 3.1 , it is easy to complete the proof of Theorem 2.2 .

Proof of Theorem 2.2: For $i \in N$, we choose elements $a_{i} \in V_{i}$ that are the best for Player 2 and the worst for Player 3 , so $a_{1}=(1-\delta \bar{b}, \delta \bar{b}, 0), a_{2}=(\delta \underline{b}, 1-\delta \underline{b}, 0)$, and $a_{3}=(0, \delta \bar{b}, 1-\delta \bar{b})$. Now let $s$ be a strategy profile that satisfies the conditions of Theorem 3.1. Since

$$
\begin{aligned}
\underline{b} & =\frac{1}{3}(1-\delta \bar{b}), \\
\bar{b} & =\frac{1}{3}(1-\delta \underline{b})+\frac{2}{3} \delta \bar{b},
\end{aligned}
$$

we find that the strategy profile $s$ yields a payoff of $\bar{b}$ for Player 2 and a payoff of $\underline{b}$ for Player 3.

In any SPE in stationary strategies, the proposer obtains a payoff of $1-\delta / 3$ in period 0 and one of the responders receives a payoff of $\delta / 3$. The stationary equilibrium is usually taken as a theoretical benchmark in the experimental literature. In this literature, substantial deviations from this benchmark are found. McKelvey (1991) describes an experiment with a discrete version of the BF model. Compared to the stationary prediction, proposers usually offer too much to the responding players. Diermeier and Morton (2006) consider a finitely repeated version of the BF model. Similar to McKelvey (1991), Diermeier and Morton (2006) find little support for the predictions of the stationary equilibrium. First, in 
one third of the cases a positive amount was proposed to all players, not just to the members of the minimal winning coalition. Moreover, proposers consistently offer too much to other coalition members. Both McKelvey (1991) and Diermeier and Morton (2006) report the use of non-stationary strategies. We verify to what extent the findings in the experimental literature are consistent with the non-stationary subgame perfect equilibria as found in Theorem 3.1. We refer to the responder who is offered the higher share in the cake as Player $j$, and to the other responder as Player $k$.

Fréchette, Kagel, and Morelli (2005) provide experimental evidence for the underrealization of proposer power and the generosity shown to voters outside the minimal winning coalition for the BF model with $\delta=0.5$ and $\delta=1$. They consider both treatments with experienced and treatments with inexperienced players.

When $\delta=1,39 \%$ of the proposals for inexperienced players do not correspond to the formation of a minimum winning coalition, a number that is still equal to $23 \%$ for experienced players. Even perfectly egalitarian proposals are observed in more than $5 \%$ of the cases. A perfectly egalitarian proposal is consistent with subgame perfect equilibrium when $1-\delta \bar{b} \leq 1 / 3$, which is equivalent to $\delta \geq(21-3 \sqrt{41}) / 2 \approx 0.895$. The average share of the proposer for accepted offers is 0.51 for inexperienced players and 0.52 for experienced players, considerably lower than the stationary equilibrium prediction of 0.67. The average share of Player $j$ is 0.43 for inexperienced players and 0.45 for experienced players, considerably more than the stationary equilibrium prediction of 0.33 . Since we have shown that any proposal and any payoff is possible in an SPE for discount factors tending to one, these results would not contradict SPE.

When $\delta=0.5,57 \%$ of the proposals for inexperienced players do not correspond to the formation of a minimum winning coalition, a number that is still equal to $23 \%$ for experienced players. The average share of the proposer for accepted offers is 0.50 for inexperienced players and 0.59 for experienced players, considerably lower than the stationary equilibrium prediction of 0.83 . The average share of Player $j$ is 0.41 for inexperienced players and 0.39 for experienced players, considerably more than the stationary equilibrium prediction of 0.17. Our results predict a payoff to the proposer in the range from $1-\delta \bar{b}=0.78$ to $1-\delta \underline{b}=0.87$. The payoff to the player who receives the higher share is predicted to be in the range from $\delta \underline{b}=0.13$ to $\delta \bar{b}=0.22$. The payoff to the player who receives the lower share is predicted to be in the range from 0 to $\delta \bar{b} / 2=0.11$. For $\delta=0.5$ the experimental findings are not even consistent with SPE. This is not so surprising, since for such low values of the discount factor, the potential influence of punishment is quite limited. Fréchette, Kagel, and Morelli (2005) offer the reluctance of coalition partners to accept offers much below $1 / 3$ as an explanation of these findings.

Kagel, Sung, and Winter (2010) consider a treatment corresponding to the BF model 
with a discount factor $\delta=0.95$. They report that $40.8 \%$ of the proposals do not correspond to the formation of a minimum winning coalition. For accepted offers, the average share of the proposer is 0.458 and for Player $j$ it is 0.44 . Very similar results are found in Breitmoser and Tan (2010), who also study the case $\delta=0.95$. The sample estimates are 0.445 to the proposer, 0.359 to Player $j$, and 0.172 to Player $k$. The stationary equilibrium predicts a payoff of 0.683 to the proposer, 0.317 to Player $j$, and 0 to Player $k$. Our results predict a payoff to the proposer in the range from $1-\delta \bar{b}=0.188$ to $1-\delta \underline{b}=0.938$. The payoff to the player who receives the higher share is predicted to be in the range from $\delta \underline{b}=0.063$ to $\delta \bar{b}=0.813$. The payoff to the player who receives the lower share is predicted to be in the range from 0 to $\delta \bar{b}=0.406$.

Miller and Vanberg (2013) compare the costs of reaching agreement under majority and unanimity rule in the context of an experimental bargaining game with $\delta=0.9$. They find patterns very similar to those reported in the previous literature. Proposers demand a higher share than they allocate to non-proposers, but the difference is far from the equilibrium prediction. Interestingly, approximately half of the proposals in the first period are three-way equal splits and only one out of five allocates 0 to one of the non-proposers. We have shown that three-way equal splits are SPE payoffs for $\delta \geq 0.84$.

\section{Equilibria with one-period delay}

The experimental literature shows that one cannot rule out delay before acceptance takes place. Fréchette, Kagel, and Morelli (2005) conclude that a majority of the proposals are accepted without delay, however, delays persist until the end of the sessions. They find one or more rounds of delay for experienced subjects in $5 \%$ of the cases when $\delta=0.5$, a number increasing to $23 \%$ when $\delta=1$. For inexperienced subjects, one or more rounds of delay occur in $11 \%$ of the cases when $\delta=0.5$ and in $32 \%$ of the cases when $\delta=1$. Kagel, Sung, and Winter (2010) report one or more rounds of delay in $28 \%$ of the cases when $\delta=0.95$. Miller and Vanberg (2013) consider the case where $\delta=0.9$ and find one or more rounds of delay before acceptance takes place in $25 \%$ of the cases.

In this section we derive the necessary and sufficient conditions to have exactly one period of delay before acceptance takes place in an SPE. A strategy profile $s$ is said to have one-period delay if, irrespectively of the moves of nature, the proposal in period 0 is rejected by both responders, and the proposal in period 1 is accepted by at least one responder. More precisely, the strategy profile $s$ has one-period delay if for all permutations $\pi^{0}$ and $\pi^{1}$ the proposal $s_{\pi^{0}(1)}\left(\pi^{0}\right)$ is rejected by both Players $\pi^{0}(2)$ and $\pi^{0}(3)$, while the proposal $s_{\pi^{1}(1)}\left(\pi^{0}, s_{\pi^{0}(1)}\left(\pi^{0}\right), \pi^{1}\right)$ is accepted by $\pi^{1}(2)$ or $\pi^{1}(3)$. 
Theorem 4.1 There exists an SPE with one-period delay if and only if $\delta \geq 6-3 \sqrt{3} \approx$ 0.804 .

The condition $\delta \geq 6-3 \sqrt{3}$ is derived from the inequality $1-\delta \bar{b} \leq \delta \bar{b}$, which expresses that the minimum equilibrium payoff conditional on being the proposer should be less than or equal to the discounted maximum unconditional equilibrium payoff. We first prove the only if part of Theorem 4.1.

Claim 4.2 If there is an SPE with one-period delay, then $\delta \geq 6-3 \sqrt{3}$.

Proof: Let $s$ be an SPE with one-period delay. Without loss of generality, assume that $\pi^{0}=(1,2,3)$. Conditional on $\pi^{0}$, the payoff to Player 1 is at most $\delta \bar{u}$. On the other hand, for each $\epsilon>0$, the proposal $(1-\delta \bar{u}-\epsilon, \delta \bar{u}+\epsilon, 0)$ is accepted by Claim 2.4. Since $s$ is an SPE, we must have $1-\delta \bar{u}-\epsilon \leq \delta \bar{u}$ for each $\epsilon>0$, and hence $1-\delta \bar{u} \leq \delta \bar{u}$. Since $\bar{u}=\bar{b}$ by Theorem 2.2 , we find $1-\delta \bar{b} \leq \delta \bar{b}$, so

$$
1-\frac{3 \delta-\delta^{2}}{9 \delta-6 \delta^{2}-\delta^{3}} \leq \frac{3 \delta-\delta^{2}}{9 \delta-6 \delta^{2}-\delta^{3}}
$$

Rearranging terms, we find that $\delta \geq 6-3 \sqrt{3}$.

To prove the if part of Theorem 4.1, we construct an SPE $\widehat{s}$ such that on the equilibrium path of play the proposer $\pi^{0}(1)$ in period 0 demands the entire surplus, his proposal is rejected, and the proposal in period 1 by Player $\pi^{1}(1)$ is accepted leading to an expected payoff of $\delta \bar{b}$ to Player $\pi^{0}(1)$. Any deviation by Player $\pi^{0}(1)$ results in a payoff of at most $1-\delta \bar{b}$. Under the assumption that $1-\delta \bar{b} \leq \delta \bar{b}$ such a deviation is not profitable.

Let $\tau^{1}$ be the SPE constructed in Theorem 3.1 with $a_{1}=(1-\delta \underline{b}, \delta \underline{b}, 0), a_{2}=(\delta \bar{b}, 1-$ $\delta \bar{b}, 0)$, and $a_{3}=(\delta \bar{b}, 0,1-\delta \bar{b})$. Strategy profile $\tau^{1}$ gives an expected payoff of $\bar{b}$ to Player 1. Similarly, let $\tau^{2}$ be the SPE provided by Theorem 3.1 with $a_{1}=(1-\delta \bar{b}, \delta \bar{b}, 0), a_{2}=$ $(0,1-\delta \underline{b}, \delta \underline{b})$, and $a_{3}=(0, \delta \bar{b}, 1-\delta \bar{b})$, and let $\tau^{3}$ be the SPE with $a_{1}=(1-\delta \bar{b}, 0, \delta \bar{b})$, $a_{2}=(0,1-\delta \bar{b}, \delta \bar{b})$, and $a_{3}=(0, \delta \underline{b}, 1-\delta \underline{b})$. Strategy profile $\tau^{2}$ gives an expected payoff of $\bar{b}$ to Player 2 and $\tau^{3}$ gives an expected payoff of $\bar{b}$ to Player 3 .

Let $\gamma$ be the SPE delivered by Theorem 3.1 with $a_{1}=(1-\delta \bar{b}, \delta \bar{b}, 0), a_{2}=(0,1-\delta \bar{b}, \delta \bar{b})$, and $a_{3}=(\delta \bar{b}, 0,1-\delta \bar{b})$. The payoffs corresponding to $\gamma$ are $(1 / 3,1 / 3,1 / 3)$.

The one-period delay strategy profile $\widehat{s}$ is defined by means of the strategy profiles $\tau^{1}, \tau^{2}, \tau^{3}$, and $\gamma$. The initial proposer $\pi^{0}(1)$ proposes to keep the entire surplus, so proposes the unit vector $e_{\pi^{0}(1)}$, a proposal which is rejected. For histories where the initial proposer has deviated, $\widehat{s}$ is defined to be equal to $\gamma$. Otherwise, starting in period $1, \widehat{s}$ is defined to be equal to $\tau^{\pi^{0}(1)}$, where the period 0 history is deleted before applying $\tau^{\pi^{0}(1)}$. Whomever 
is the initial proposer in Period 0 is maximally rewarded in Period 1 . More precisely, we have the following definitions.

For period $t=0$, we define

$$
\begin{aligned}
& \widehat{s}_{\pi^{0}(1)}\left(\pi^{0}\right) \quad=e_{\pi^{0}(1)}, \\
& \widehat{s}_{\pi^{0}(2)}\left(\pi^{0}, e_{\pi^{0}(1)}\right)=\widehat{s}_{\pi^{0}(3)}\left(\pi^{0}, e_{\pi^{0}(1)}\right)=\mathrm{r}, \\
& \widehat{s}_{\pi^{0}(2)}\left(\pi^{0}, x^{0}\right) \quad=\gamma_{\pi^{0}(2)}\left(\pi^{0}, x^{0}\right), \quad \text { if } x^{0} \neq e_{\pi^{0}(1)} \text {, } \\
& \widehat{s}_{\pi^{0}(3)}\left(\pi^{0}, x^{0}\right) \quad=\gamma_{\pi^{0}(3)}\left(\pi^{0}, x^{0}\right), \quad \text { if } x^{0} \neq e_{\pi^{0}(1)} \text {. }
\end{aligned}
$$

When the proposal $e_{\pi^{0}(1)}$ is made in period 0 , we define $\widehat{s}$ for histories in period $t \geq 1$ by

$$
\begin{aligned}
& \widehat{s}_{\pi^{t}(1)}\left(\pi^{0}, e_{\pi^{0}(1)}, \pi^{1}, x^{1}, \ldots, \pi^{t}\right)=\tau_{\pi^{t}(1)}^{\pi^{0}(1)}\left(\pi^{1}, x^{1}, \ldots, \pi^{t}\right), \\
& \widehat{s}_{\pi^{t}(2)}\left(\pi^{0}, e_{\pi^{0}(1)}, \pi^{1}, x^{1}, \ldots, \pi^{t}, x^{t}\right)=\tau_{\pi^{t}(2)}^{\pi^{0}(1)}\left(\pi^{1}, x^{1} \ldots, \pi^{t}, x^{t}\right), \\
& \widehat{s}_{\pi^{t}(3)}\left(\pi^{0}, e_{\pi^{0}(1)}, \pi^{1}, x^{1}, \ldots, \pi^{t}, x^{t}\right)=\tau_{\pi^{t}(3)}^{\pi^{0}(1)}\left(\pi^{1}, x^{1} \ldots, \pi^{t}, x^{t}\right) .
\end{aligned}
$$

When the proposal $x^{0}$ made in period 0 is not equal to $e_{\pi^{0}(1)}$, we define $\widehat{s}$ for histories in period $t \geq 1$ by

$$
\begin{aligned}
& \widehat{s}_{\pi^{t}(1)}\left(\pi^{0}, x^{0}, \ldots, \pi^{t}\right)=\gamma_{\pi^{t}(1)}\left(\pi^{0}, x^{0}, \ldots, \pi^{t}\right), \\
& \widehat{s}_{\pi^{t}(2)}\left(\pi^{0}, x^{0}, \ldots, \pi^{t}, x^{t}\right)=\gamma_{\pi^{t}(2)}\left(\pi^{0}, x^{0}, \ldots, \pi^{t}, x^{t}\right), \\
& \widehat{s}_{\pi^{t}(3)}\left(\pi^{0}, x^{0}, \ldots, \pi^{t}, x^{t}\right)=\gamma_{\pi^{t}(3)}\left(\pi^{0}, x^{0}, \ldots, \pi^{t}, x^{t}\right) .
\end{aligned}
$$

Let $\ell \in\{0, \ldots, t\}$. For a history $h$ in period $t$ we define "the tail" $h^{-\ell}$ of $h$ obtained by deleting the first $\ell$ periods from $h$. More precisely, for $h=\left(\pi^{0}, x^{0}, \ldots, \pi^{t}\right)$ we define $h^{-\ell}=\left(\pi^{\ell}, x^{\ell}, \ldots, \pi^{t}\right)$, and for $h=\left(\pi^{0}, x^{0}, \ldots, \pi^{t}, x^{t}\right)$ we define $h^{-\ell}=\left(\pi^{\ell}, x^{\ell}, \ldots, \pi^{t}, x^{t}\right)$. In particular $h^{-0}=h$.

The claim below makes an almost trivial observation: Assume that the strategy profile $s$ is an SPE of $\Gamma$. Assume furthermore that the strategy profile $\widehat{s}$ is such that, as soon as some given history $h$ in period $\ell$ has been reached, the strategy profile $\widehat{s}$ is equal to $s$, where $s$ is applied after deleting the first $\ell$ periods from the history. Then $\widehat{s}$ is subgame perfect in the subgame starting at $h$. For a history $h \in H$, we denote the player who acts at $h$ by $\iota(h)$. For history $h, h^{\prime}$, we write $h \geq h^{\prime}$ if $h=h^{\prime}$ or the history $h$ extends the history $h^{\prime}$.

Claim 4.3 Let $s$ be an SPE of $\Gamma$ and let $h^{\prime}$ be a history in period $\ell$. Let the strategy profile $\widehat{s}$ be such that $\widehat{s}_{\iota(h)}(h)=s_{\iota(h)}\left(h^{-\ell}\right)$ for each history $h \geq h^{\prime}$. Then $\widehat{s}$ is subgame perfect in the subgame starting at $h^{\prime}$. 
Proof: The subgame starting at history $h$ is isomorphic to the one starting at $h^{-\ell}$. Hence, if there would be a profitable one-shot deviation from $\widehat{s}$ at $h$, there would be one from $s$ at $h^{-\ell}$.

As it is clear that $\widehat{s}$ is a strategy profile with one-period delay, the if part of Theorem 4.1 follows immediately from the following claim.

Claim 4.4 If $\delta \geq 6-3 \sqrt{3}$, then $\widehat{s}$ is an $S P E$.

Proof: Consider the proposer history $\left(\pi^{0}\right)$. Under the strategy profile $\widehat{s}$, Player $\pi^{0}(1)$ makes the proposal $e_{\pi^{0}(1)}$, a proposal that is followed by a rejection, and the acceptance of the proposal $\widehat{s}_{\pi^{1}(1)}\left(\pi^{0}, e_{\pi^{0}(1)}, \pi^{1}\right)$ next period, leading to an expected payoff of $\delta \bar{b}$ to Player $\pi^{0}(1)$. We argue that Player $\pi^{0}(1)$ does not have a profitable one-shot deviation from $\widehat{s}_{\pi^{0}(1)}$ at $\left(\pi^{0}\right)$. Consider a one-shot deviation to $x^{0} \neq e_{\pi^{0}(1)}$ by Player $\pi^{0}(1)$. For histories $h \geq\left(\pi^{0}, x^{0}\right)$, it holds that $\widehat{s}_{\iota(h)}(h)=\gamma_{\iota(h)}(h)$.

At history $\left(\pi^{0}\right)$, the strategy profile $\gamma$ requires that the proposal $\gamma_{\pi^{0}(1)}\left(\pi^{0}\right)$ is made and accepted, which gives Player $\pi^{0}(1)$ a utility equal to $1-\delta \bar{b}$. Since $\gamma$ is subgame perfect, Player $\pi^{0}(1)$ does not have a profitable one-shot deviation from $\gamma$, so under $\gamma$, the expected payoff of Player $\pi^{0}(1)$ following any proposal $y$ is less than or equal to $1-\delta \bar{b}$. Since for histories $h \geq\left(\pi^{0}, x^{0}\right)$, it holds that $\widehat{s}_{\iota(h)}(h)=\gamma_{\iota(h)}(h)$, we find that under $\widehat{s}$, a one-shot deviation to $x^{0} \neq e_{\pi^{0}(1)}$ by Player $\pi^{0}(1)$ gives him an expected payoff less than or equal to $1-\delta \bar{b}$. Since $\delta \geq 6-3 \sqrt{3}$, we have that $1-\delta \bar{b} \leq \delta \bar{b}$, so the one-shot deviation to $x^{0}$ is not profitable.

Consider the responder history $\left(\pi^{0}, e_{\pi^{0}(1)}\right)$. According to $\widehat{s}$, both responders reject the proposal $e_{\pi^{0}(1)}$. Accepting the proposal gives a responder a payoff equal to 0 and is clearly not a profitable deviation from $\widehat{s}$.

For all other histories, the one-shot deviation property follows from Claim 4.3. Indeed, consider $x^{0} \neq e_{\pi^{0}(1)}$. For histories $h \geq\left(\pi^{0}, x^{0}\right)$, the one-shot deviation property follows from Claim 4.3 with $h_{0}=\left(\pi^{0}, x^{0}\right), \ell=0$, and $s=\gamma$. For histories $h \geq\left(\pi^{0}, e_{\pi^{0}(1)}, \pi^{1}\right)$, the one-shot deviation property follows from Claim 4.3 with $h_{0}=\left(\pi^{0}, e_{\pi^{0}(1)}, \pi^{1}\right), \ell=1$, and $s=\tau^{\pi^{0}(1)}$.

\section{Arbitrarily long delay}

In this section we show that any finite delay is compatible with an SPE, provided that the players are patient enough. By analogy with the case of one-period delay, we say that the 
strategy profile $s$ has $m$-period delay if, irrespective of the moves by nature, the proposals in periods $0, \ldots, m-1$ are rejected, and the proposal in period $m$ is accepted.

Theorem 5.1 Consider any $m \geq 1$. Then there exists a strategy profile $\widetilde{s}$ with $m$-period delay and a discount factor $\widetilde{\delta}<1$ such that $\widetilde{s}$ is an SPE whenever $\delta \geq \widetilde{\delta}$.

We let $\widetilde{\delta}$ be the unique value of $\delta$ that solves the equation

$$
\frac{\delta^{m}}{3}=1-\delta \frac{3-\delta}{9-6 \delta-\delta^{2}} .
$$

Notice that the right-hand side of this equation is exactly $1-\delta \bar{b}$. This is a decreasing function of $\delta$ with value 1 at $\delta=0$ and value 0 at $\delta=1$. Hence the solution exists and is unique. Moreover, for each $\delta>\widetilde{\delta}$ it holds that $\delta^{m} / 3>1-\delta \bar{b}$.

Since

$$
0=\widetilde{\delta}^{m+2}+6 \widetilde{\delta}^{m+1}-9 \widetilde{\delta}^{m}-27 \widetilde{\delta}+27 \leq \widetilde{\delta}^{m}+6 \widetilde{\delta}^{m}-9 \widetilde{\delta}^{m}-27 \widetilde{\delta}^{m}+27=-29 \widetilde{\delta}^{m}+27,
$$

it follows that $\widetilde{\delta} \leq(27 / 29)^{1 / m}$.

For $\delta \geq \widetilde{\delta}$, the vector $\left(\delta^{m} / 3, \delta^{m} / 3, \delta^{m} / 3\right)$ belongs to the sets $V_{1}, V_{2}$, and $V_{3}$, since $\delta^{m} / 3 \geq 1-\delta \bar{b}$ and $1-\delta \bar{b} \geq \delta \underline{b}$. We let $\gamma$ be the strategy profile provided by Theorem 3.1 with $a_{1}=a_{2}=a_{3}=\left(\delta^{m} / 3, \delta^{m} / 3, \delta^{m} / 3\right)$. The vector $(1 / 3,1 / 3,1 / 3)$ also belongs to the sets $V_{1}, V_{2}$, and $V_{3}$. Let $\tau$ be the strategy profile provided by Theorem 3.1 with $a_{1}=a_{2}=a_{3}=(1 / 3,1 / 3,1 / 3)$.

We construct a strategy profile $\widetilde{s}$ such that on the equilibrium path of play in periods $0, \ldots, m-1$ the proposers demand the entire surplus and their proposals are rejected. If period $m$ is reached without deviations, the history of the preceding periods is deleted and as of period $m$ the strategy profile $\tau$ is followed. In particular, on the equilibrium path of play, the equal split is proposed and accepted in period $m$. However, as soon as a proposer deviates in some period $\ell<m$, the history of the preceding periods is deleted, and the strategy profile $\gamma$ is followed.

We partition the set $H$ of histories into pairwise disjoint sets $D_{-1}, D_{0}, \ldots, D_{m}$ as follows:

$$
D_{-1}=\left\{h \in H \mid\left(\pi^{0}, e_{\pi^{0}(1)}, \ldots, \pi^{m-1}, e_{\pi^{m-1}(1)}\right) \geq h\right\}
$$

for $0 \leq \ell<m$,

$$
D_{\ell}=\left\{h \in H \mid \exists x^{\ell} \in V \backslash\left\{e_{\pi^{\ell}(1)}\right\} \text { such that } h \geq\left(\pi^{0}, e_{\pi^{0}(1)}, \ldots, \pi^{\ell-1}, e_{\pi^{\ell-1}(1)}, \pi^{\ell}, x^{\ell}\right)\right\},
$$

and

$$
D_{m}=\left\{h \in H \mid h \geq\left(\pi^{0}, e_{\pi^{0}(1)}, \ldots, \pi^{m-1}, e_{\pi^{m-1}(1)}, \pi^{m}\right)\right\} .
$$


We define the strategy profile $\widetilde{s}$ as follows:

$$
\begin{aligned}
\widetilde{s}_{\pi^{\ell}(1)}\left(\pi^{0}, e_{\pi^{0}(1)}, \ldots, \pi^{\ell-1}, e_{\pi^{\ell-1}(1)}, \pi^{\ell}\right) & =e_{\pi^{\ell}(1)}, & & 0 \leq \ell<m, \\
\widetilde{s}_{\pi^{\ell}(2)}\left(\pi^{0}, e_{\pi^{0}(1)}, \ldots, \pi^{\ell}, e_{\pi^{\ell}(1)}\right) & =\mathrm{r}, & & 0 \leq \ell<m, \\
\widetilde{s}_{\pi^{\ell}(3)}\left(\pi^{0}, e_{\pi^{0}(1)}, \ldots, \pi^{\ell}, e_{\pi^{\ell}(1)}\right) & =\mathrm{r}, & & 0 \leq \ell<m, \\
\widetilde{s}_{\iota(h)}(h) & =\tau_{\iota(h)}\left(h^{-m}\right), & & h \in D_{m}, \\
\widetilde{s}_{\iota(h)}(h) & =\gamma_{\iota(h)}\left(h^{-\ell}\right), & & h \in D_{\ell}, 0 \leq \ell<m .
\end{aligned}
$$

Equations (5.1)-(5.3) specify $\widetilde{s}$ on the set of histories $D_{-1}$, Equation (5.4) on $D_{m}$, and Equation (5.5) on $D_{\ell}$ for $\ell \in\{0, \ldots, m-1\}$.

From Equation (5.4) we get

$$
\begin{aligned}
\widetilde{s}_{\pi^{m}(1)}\left(\pi^{0}, e_{\pi^{0}(1)}, \ldots, \pi^{m-1}, e_{\pi^{m-1}(1)}, \pi^{m}\right) & =\tau_{\pi^{m}(1)}\left(\pi^{m}\right)=\left(\frac{1}{3}, \frac{1}{3}, \frac{1}{3}\right), \\
\widetilde{s}_{\pi^{m}(2)}\left(\pi^{0}, e_{\pi^{0}(1)}, \ldots, \pi^{m-1}, e_{\pi^{m-1}(1)}, \pi^{m},\left(\frac{1}{3}, \frac{1}{3}, \frac{1}{3}\right)\right) & =\tau_{\pi^{m}(2)}\left(\pi^{m},\left(\frac{1}{3}, \frac{1}{3}, \frac{1}{3}\right)\right)=\mathrm{a}, \\
\widetilde{s}_{\pi^{m}(3)}\left(\pi^{0}, e_{\pi^{0}(1)}, \ldots, \pi^{m-1}, e_{\pi^{m-1}(1)}, \pi^{m},\left(\frac{1}{3}, \frac{1}{3}, \frac{1}{3}\right)\right) & =\tau_{\pi^{m}(3)}\left(\pi^{m},\left(\frac{1}{3}, \frac{1}{3}, \frac{1}{3}\right)\right)=\mathrm{a} .
\end{aligned}
$$

Equations (5.1)-(5.3) and (5.6)-(5.8) specify the equilibrium path of play under $\widetilde{s}$ : The proposals $e_{\pi^{0}(1)}, \ldots, e_{\pi^{m-1}(1)}$ are made and rejected, followed by the proposal $(1 / 3,1 / 3,1 / 3)$, which is accepted. Thus the strategy profile $\widetilde{s}$ has $m$-period delay. All players receive a payoff of $\delta^{m} / 3$. Equation (5.4) specifies the play of the game as of period $m$, provided that no deviations have occurred before period $m$. Equation (5.5) specifies the continuation play following a deviation in period $\ell<m$.

In case $m=1$, the construction above resembles that in the previous section. One difference is that here the play following histories of the form $\left(\pi^{0}, e_{\pi^{0}(1)}, \pi^{1}\right)$ as defined by Equation (5.4) is independent of the permutation $\pi^{0}$, whereas in the preceding section it does depend on $\pi^{0}(1)$.

Claim 5.2 If $\delta \geq \widetilde{\delta}$, then $\widetilde{s}$ is an SPE with $m$-period delay.

Proof: We show that there are no profitable one-shot deviations from $\widetilde{s}$.

For some $\ell \in\{0, \ldots, m-1\}$, consider the proposer history $\left(\pi^{0}, e_{\pi^{0}(1)}, \ldots, \pi^{\ell-1}, e_{\pi^{\ell-1}(1)}, \pi^{\ell}\right)$. Under the strategy profile $\widetilde{s}$, Player $\pi^{\ell}(1)$ receives a payoff of $\delta^{m} / 3$. Consider a one-shot deviation to $x^{\ell} \neq e_{\pi^{\ell}(1)}$ by Player $\pi^{\ell}(1)$. For histories $h \geq\left(\pi^{0}, e_{\pi^{0}(1)}, \ldots, \pi^{\ell-1}, e_{\pi^{\ell-1}(1)}, \pi^{\ell}, x^{\ell}\right)$, it holds that $\widetilde{s}_{\iota(h)}(h)=\gamma_{\iota(h)}\left(h^{-\ell}\right)$.

At history $\left(\pi^{\ell}\right)$, the strategy profile $\gamma$ requires that the proposal $\left(\delta^{m} / 3, \delta^{m} / 3, \delta^{m} / 3\right)$ is made and accepted. Since $\gamma$ is subgame perfect for $\delta \geq \widetilde{\delta}$, Player $\pi^{\ell}(1)$ does not have a profitable one-shot deviation from $\gamma$, so under $\gamma$, the expected payoff of Player $\pi^{\ell}(1)$ following any proposal $y$ is less than or equal to $\delta^{m} / 3$. Since for histories $h \geq$ $\left(\pi^{0}, e_{\pi^{0}(1)}, \ldots, \pi^{\ell-1}, e_{\pi^{\ell-1}(1)}, \pi^{\ell}, x^{\ell}\right)$, it holds that $\widetilde{s}_{\iota(h)}(h)=\gamma_{\iota(h)}\left(h^{-\ell}\right)$, we find that under $\widetilde{s}$, 
a one-shot deviation to $x^{\ell} \neq e_{\pi^{\ell}(1)}$ by Player $\pi^{\ell}(1)$ gives him an expected payoff less than or equal to $\delta^{\ell} \delta^{m} / 3$. The one-shot deviation is therefore not profitable.

For some $\ell \in\{0, \ldots, m-1\}$, consider the responder history $h=\left(\pi^{0}, e_{\pi^{0}(1)}, \ldots, \pi^{\ell}, e_{\pi^{\ell}(1)}\right)$. According to $\widetilde{s}$, both responders reject the proposal $e_{\pi^{\ell}(1)}$. Accepting the proposal gives a responder a payoff equal to 0 and is clearly not a profitable deviation from $\widetilde{s}$.

For histories in $D_{0}, \ldots, D_{m}$, the one-shot deviation property follows by an application of Claim 4.3.

\section{Conclusion}

In this paper we consider the Baron and Ferejohn (1989) model, where three players use majority voting to divide a surplus in the presence of discounting. We give a complete characterization of the set of subgame perfect equilibrium payoffs for any value of the discount factor. Although the usual statement of the folk theorem does not hold in this setting, we show that any given vector in the interior of the set of feasible payoffs is a subgame perfect equilibrium outcome for a sufficiently high value of the discount factor.

Many papers in the experimental literature use the subgame perfect equilibrium in stationary strategies as a benchmark and conclude that this equilibrium is not supported by the data. The experimental findings are however not in contradiction to the predictions of subgame perfect equilibrium once the stationarity assumption is dropped and the discount factor is sufficiently high. Experimental evidence for a discount factor as low as $1 / 2$ cannot be explained by non-stationary subgame perfect equilibria. Experimental papers also find significant amounts of delay before an acceptance takes place, where the delay probability increases with the discount factor. We provide necessary and sufficient conditions on the discount factor to have one period of delay, and we construct subgame perfect equilibria where arbitrary long delay occurs whenever the discount factor is sufficiently high. 


\section{Appendix: The proof of Theorem 3.1}

Theorem 3.1 is proven separately in two subsections for the case $\delta \leq 3 / 5$ and for the case $\delta>3 / 5$. In both cases the strategy profile $s$ is constructed along the same lines.

We introduce some additional notation. Consider a responder history $\left(\pi^{0}, x^{0}, \ldots, \pi^{t}, x^{t}\right)$. We denote the proposer $\pi^{t}(1)$ in period $t$ by $p^{t}$. The responders in period $t$ are denoted by $j^{t}$ and $k^{t}$, respectively, where $j^{t}$ is chosen such that his share in the cake weakly exceeds that of player $k^{t}$, and $j^{t}=\pi^{t}(2)$ in case both shares are equal, so

$$
j^{t}= \begin{cases}\pi^{t}(2), & \text { if }\left[x_{\pi^{t}(2)}^{t} \geq x_{\pi^{t}(3)}^{t}\right] \\ \pi^{t}(3), & \text { if }\left[x_{\pi^{t}(3)}^{t}>x_{\pi^{t}(2)}^{t}\right] .\end{cases}
$$

To avoid excessive notation, we write $x_{j}^{t}$ and $x_{k}^{t}$ instead of $x_{j^{t}}^{t}$ and $x_{k^{t}}^{t}$, respectively. A proposer history $h=\left(\pi^{0}, x^{0}, \ldots, \pi^{t}, x^{t}, \pi^{t+1}\right)$ uniquely defines the finite sequence

$$
h^{*}=\left(p^{0}, x^{0}, j^{0}, k^{0}, \ldots, p^{t}, x^{t}, j^{t}, k^{t}, p^{t+1}\right) .
$$

We define the strategy for the proposer using sequences of this form, leading to functions $\rho^{0}, \rho^{1}, \ldots$ These functions are defined recursively as follows. Set $\rho^{0}\left(p^{0}\right)=a_{p^{0}}$ and for each $t \geq 0$ let

$$
\rho^{t+1}\left(p^{0}, \ldots, p^{t}, x^{t}, j^{t}, k^{t}, p^{t+1}\right)=f\left(p^{t}, x^{t}, j^{t}, k^{t}, p^{t+1}, \rho^{t}\left(p^{0}, \ldots, p^{t}\right)\right),
$$

where each of the subsections below uses a particular specification for the function $f$. With a minor abuse of notation we write $\rho^{t}(h)$ rather than $\rho^{t}\left(h^{*}\right)$.

\section{The proof of Theorem 3.1 for $\delta \leq 3 / 5$}

Throughout this subsection it is assumed that $\delta \leq 3 / 5$. The function $f$ is defined in Table 1 and Table 2. If after the proposer history $h$ in period $t$ the proposer complies with his strategy and makes a proposal $x^{t}=\rho^{t}(h)$, then the proposals in period $t+1$ are determined by Table 1 . If the proposer deviates from his strategy and makes a proposal $x^{t} \neq \rho^{t}(h)$, then the proposals in period $t+1$ are determined by Table 2 . The first three columns of the tables list the six possible values for $\left(p^{t}, j^{t}, k^{t}\right)$, and the last three columns specify $f\left(p^{t}, x^{t}, j^{t}, k^{t}, p^{t+1}, y^{t}\right)$ for each of the three possible values of $p^{t+1}$.

We define the strategy profile $s$ as follows:

1. For each $t \geq 0$, for each history $h=\left(\pi^{0}, x^{0}, \ldots, \pi^{t}\right)$, Player $p^{t}$ proposes $\rho^{t}(h)$.

2. After history $h=\left(\pi^{0}, x^{0}, \ldots, \pi^{t}, x^{t}\right)$, Player $j^{t}$ accepts $x^{t}$ if 


\begin{tabular}{|c|c|c|c|c|c|}
\hline$p^{t}$ & $j^{t}$ & $k^{t}$ & $p^{t+1}=1$ & $p^{t+1}=2$ & $p^{t+1}=3$ \\
\hline 1 & 2 & 3 & $(1-\delta \underline{b}, 0, \delta \underline{b})$ & $(\delta \bar{b}, 1-\delta \bar{b}, 0)$ & $(\delta \bar{b}, 0,1-\delta \bar{b})$ \\
\hline 1 & 3 & 2 & $(1-\delta \underline{b}, \delta \underline{b}, 0)$ & $(\delta \bar{b}, 1-\delta \bar{b}, 0)$ & $(\delta \bar{b}, 0,1-\delta \bar{b})$ \\
\hline 2 & 1 & 3 & $(1-\delta \bar{b}, \delta \bar{b}, 0)$ & $(0,1-\delta \underline{b}, \delta \underline{b})$ & $(0, \delta \bar{b}, 1-\delta \bar{b})$ \\
\hline 2 & 3 & 1 & $(1-\delta \bar{b}, \delta \bar{b}, 0)$ & $(\delta \underline{b}, 1-\delta \underline{b}, 0)$ & $(0, \delta \bar{b}, 1-\delta \bar{b})$ \\
\hline 3 & 1 & 2 & $(1-\delta \bar{b}, 0, \delta \bar{b})$ & $(0,1-\delta \bar{b}, \delta \bar{b})$ & $(0, \delta \underline{b}, 1-\delta \underline{b})$ \\
\hline 3 & 2 & 1 & $(1-\delta \bar{b}, 0, \delta \bar{b})$ & $(0,1-\delta \bar{b}, \delta \bar{b})$ & $(\delta \underline{b}, 0,1-\delta \underline{b})$ \\
\hline
\end{tabular}

Table 1: $f\left(p^{t}, x^{t}, j^{t}, k^{t}, p^{t+1}, x^{t}\right)$.

\begin{tabular}{|c|c|c|c|c|c|}
\hline$p^{t}$ & $j^{t}$ & $k^{t}$ & $p^{t+1}=1$ & $p^{t+1}=2$ & $p^{t+1}=3$ \\
\hline 1 & 2 & 3 & $(1-\delta \bar{b}, \delta \bar{b}, 0)$ & $(\delta \underline{b}, 1-\delta \underline{b}, 0)$ & $(0, \delta \bar{b}, 1-\delta \bar{b})$ \\
\hline 1 & 3 & 2 & $(1-\delta \bar{b}, 0, \delta \bar{b})$ & $(0,1-\delta \bar{b}, \delta \bar{b})$ & $(\delta \underline{b}, 0,1-\delta \underline{b})$ \\
\hline 2 & 1 & 3 & $(1-\delta \underline{b}, \delta \underline{b}, 0)$ & $(\delta \bar{b}, 1-\delta \bar{b}, 0)$ & $(\delta \bar{b}, 0,1-\delta \bar{b})$ \\
\hline 2 & 3 & 1 & $(1-\delta \bar{b}, 0, \delta \bar{b})$ & $(0,1-\delta \bar{b}, \delta \bar{b})$ & $(0, \delta \underline{b}, 1-\delta \underline{b})$ \\
\hline 3 & 1 & 2 & $(1-\delta \underline{b}, 0, \delta \underline{b})$ & $(\delta \bar{b}, 1-\delta \bar{b}, 0)$ & $(\delta \bar{b}, 0,1-\delta \bar{b})$ \\
\hline 3 & 2 & 1 & $(1-\delta \bar{b}, \delta \bar{b}, 0)$ & $(0,1-\delta \underline{b}, \delta \underline{b})$ & $(0, \delta \bar{b}, 1-\delta \bar{b})$ \\
\hline
\end{tabular}

Table 2: $f\left(p^{t}, x^{t}, j^{t}, k^{t}, p^{t+1}, y^{t}\right)$ if $x^{t} \neq y^{t}$.

- $x^{t}=\rho^{t}(h)$ or

- $x_{j}^{t} \geq \delta \bar{b}$

and rejects otherwise.

3. After history $h=\left(\pi^{0}, x^{0}, \ldots, \pi^{t}, x^{t}\right)$, Player $k^{t}$ accepts $x^{t}$ if

- $\left[x^{t}=\rho^{t}(h)\right.$ and $\left.x_{k}^{t} \geq \delta^{2} \underline{b} / 3+\delta \underline{b}\right]$ or

- $\left[x^{t} \neq \rho^{t}(h)\right.$ and $\left.x_{k}^{t} \geq \delta \underline{b}\right]$,

and rejects otherwise.

Figure 2 shows some key thresholds as a function of $\delta$.

The intuition behind strategy profile $s$ is as follows. Table 1 determines $\rho^{t+1}(h)$ after a history $h=\left(\pi^{0}, x^{0}, \ldots, x^{t}, \pi^{t+1}\right)$ such that $x^{t}=\rho^{t}\left(\pi^{0}, x^{0}, \ldots, \pi^{t}\right)$. In other words, it describes the proposal to be made after the equilibrium proposal was made and rejected in the previous period. According to Table 1, in period $t+1$ Player $p^{t}$ obtains the best possible expected outcome, $\frac{1}{3}(1-\delta \underline{b})+\frac{2}{3} \delta \bar{b}=\bar{b}$, as an encouragement for making the 


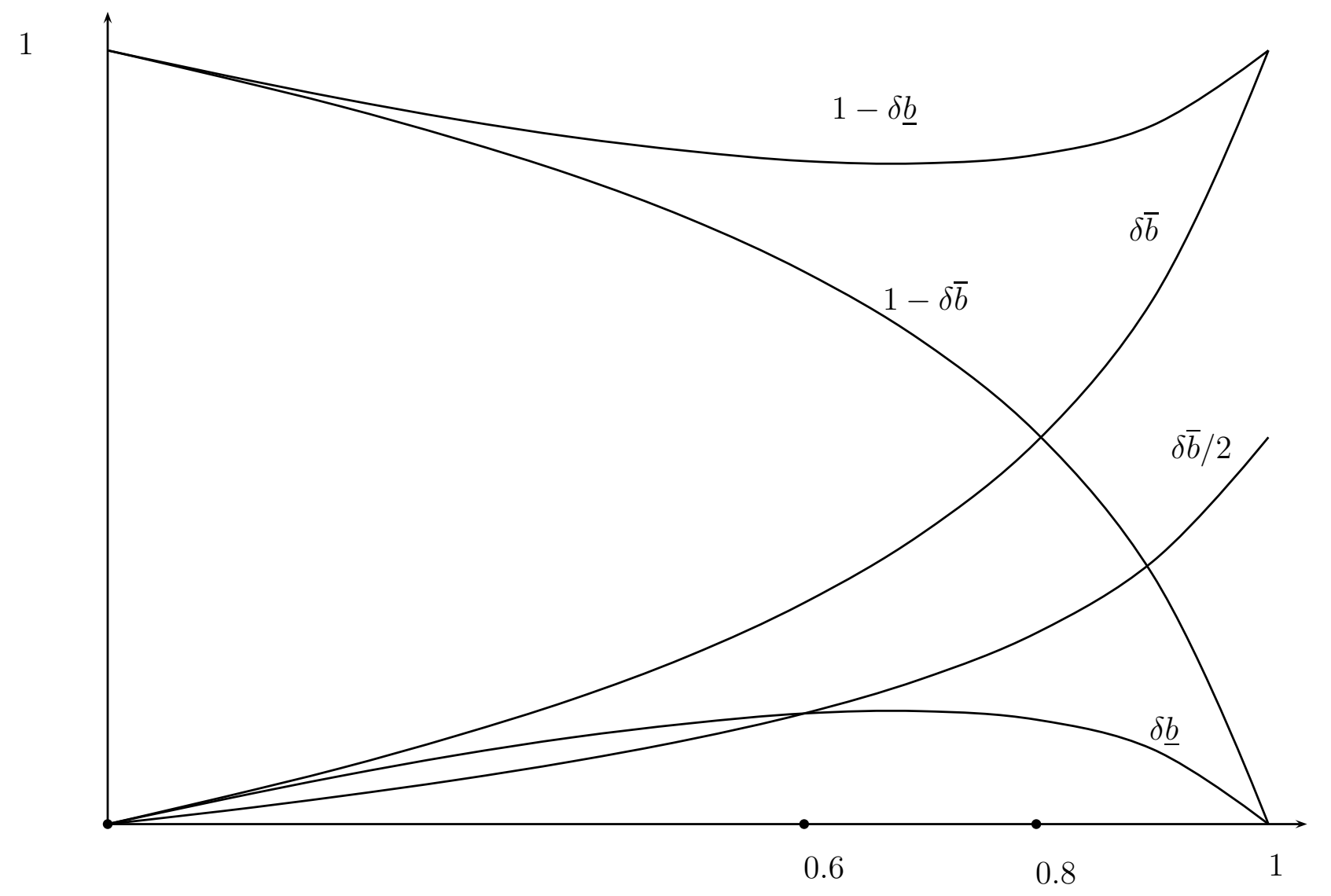

Figure 2: Key thresholds as a function of $\delta$. 
proposal described by $s$ in the previous period; Player $j^{t}$ obtains the worst possible expected outcome, $\frac{1}{3}(1-\delta \bar{b})=\underline{b}$, as a punishment for rejecting the proposal described by the strategy profile; Player $k^{t}$ receives the remainder, which is equal to $\underline{b}+\delta \underline{b} / 3$.

Table 2 determines $\rho^{t+1}(h)$ after a history $h=\left(\pi^{0}, x^{0}, \ldots, x^{t}, \pi^{t+1}\right)$ such that $x^{t} \neq$ $\rho^{t}\left(\pi^{0}, x^{0}, \ldots, \pi^{t}\right)$. In other words, it describes the proposal to be made after a deviation by the proposer in the previous period. According to Table 2, Player $p^{t}$ obtains an expected outcome of $\underline{b}+\delta \underline{b} / 3$, as a punishment for deviating in the previous period. Player $j^{t}$ obtains the best possible expected outcome of $\bar{b}$, as an encouragement for rejecting a proposal that was not described by the strategy profile. Player $k^{t}$ receives the remainder, which is equal to $\underline{b}$ in expected terms.

The proof that the strategy profile $s$ defined above is an SPE proceeds by verifying that the one-shot deviation principle is satisfied. It is well-known that the one-shot deviation principle gives the necessary and sufficient conditions for a stategy profile to be an SPE, see Fudenberg and Tirole (1991). The proof is divided into five steps. Claim A.1 states that $s$ has the "no delay property", Claim A.2 shows that after any responder history $h$ the responder who receives the lower share has no profitable one-shot deviation from $s$ at $h$. Claim A.3 show that after any responder history $h$ the responder who receives the higher share has no profitable one-shot deviation from $s$ at $h$. Claim A.4 show that proposals $x^{t} \neq \rho^{t}(h)$ which give to the proposer more than $1-\delta \bar{b}$ are rejected by both responders. And finally, Claim A.5 shows that after any proposer history $h$ the proposer has no profitable one-shot deviation from $s$ at $h$.

Claim A.1 The strategy profile $s$ has the following no delay property: if after a history $h=\left(\pi^{0}, x^{0}, \ldots, \pi^{t}\right)$ the players play according to the strategy profile $s$, then $\rho^{t}(h)$ is proposed and accepted by one of the responders and the game ends.

Proof: The claim is immediate from the definition of $s$. Indeed, the proposer proposes $x^{t}=\rho^{t}(h)$, which is accepted by Player $j^{t}$.

We now turn to the absence of profitable deviations by the player who receives the smaller share.

Claim A.2 Consider a history $h=\left(\pi^{0}, x^{0}, \ldots, \pi^{t}, x^{t}\right)$. Player $k^{t}$ has no profitable one-shot deviation from $s$ at $h$.

Proof: According to the strategy profile $s$, Player $k^{t}$ accepts $x^{t}$ if $\left[x^{t}=\rho^{t}(h)\right.$ and $x_{k}^{t} \geq$ $\left.\delta^{2} \underline{b} / 3+\delta \underline{b}\right]$ or $\left[x^{t} \neq \rho^{t}(h)\right.$ and $\left.x_{k}^{t} \geq \delta \underline{b}\right]$, and rejects otherwise. We consider four cases: 
1. $x^{t}=\rho^{t}(h)$ and $x_{k}^{t}<\delta^{2} \underline{b} / 3+\delta \underline{b}$,

2. $x^{t}=\rho^{t}(h)$ and $x_{k}^{t} \geq \delta^{2} \underline{b} / 3+\delta \underline{b}$,

3. $x^{t} \neq \rho^{t}(h)$ and $x_{k}^{t}<\delta \underline{b}$,

4. $x^{t} \neq \rho^{t}(h)$ and $x_{k}^{t} \geq \delta \underline{b}$.

The behavior of Player $k^{t}$ is irrelevant if Player $j^{t}$ responds after Player $k^{t}$ and accepts. So in the following we only need to consider the case where Player $j^{t}$ moves before Player $k^{t}$ or Player $j^{t}$ moves after Player $k^{t}$ and rejects.

Case 1. $x^{t}=\rho^{t}(h)$ and $x_{k}^{t}<\delta^{2} \underline{b} / 3+\delta \underline{b}$.

According to the strategy profile $s$, Player $k^{t}$ rejects the proposal, which leads to an expected payoff of $\delta^{t+1}(\delta \underline{b} / 3+(1-\delta \bar{b}) / 3)=\delta^{t+1}(\delta \underline{b} / 3+\underline{b})=\delta^{t}\left(\delta^{2} \underline{b} / 3+\delta \underline{b}\right)$. If Player $k^{t}$ deviates and accepts, this gives him a payoff less than $\delta^{t}\left(\delta^{2} \underline{b} / 3+\delta \underline{b}\right)$. So acceptance is not a profitable deviation.

Case 2. $x^{t}=\rho^{t}(h)$ and $x_{k}^{t} \geq \delta^{2} \underline{b} / 3+\delta \underline{b}$.

According to the strategy profile $s$, Player $k^{t}$ accepts the proposal, which leads to a payoff of at least $\delta^{t}\left(\delta^{2} \underline{b} / 3+\delta \underline{b}\right)$. If Player $k^{t}$ deviates and rejects, the expected payoff is $\delta^{t}\left(\delta^{2} \underline{b} / 3+\delta \underline{b}\right)$. So rejection is not a profitable deviation.

Case 3. $x^{t} \neq \rho^{t}(h)$ and $x_{k}^{t}<\delta \underline{b}$.

According to the strategy profile $s$, Player $k^{t}$ rejects the proposal, leading to an expected payoff of $\delta^{t+1}(1-\delta \bar{b}) / 3=\delta^{t+1} \underline{b}$. If Player $k^{t}$ deviates and accepts, it leads to a payoff less than $\delta^{t+1} \underline{b}$. So acceptance is not a profitable deviation.

Case 4. $x^{t} \neq \rho^{t}(h)$ and $x_{k}^{t} \geq \delta \underline{b}$.

According to the strategy profile $s$, Player $k^{t}$ accepts the proposal, which leads to a payoff of at least $\delta^{t+1} \underline{b}$. If Player $k^{t}$ deviates and rejects, the expected payoff is $\delta^{t+1} \underline{b}$. So rejection is not a profitable deviation.

We now turn to the absence of profitable deviations by the player who receives the higher share.

Claim A.3 Consider a history $h=\left(\pi^{0}, x^{0}, \ldots, \pi^{t}, x^{t}\right)$. Player $j^{t}$ has no profitable one-shot deviation from $s$ at $h$.

Proof: According to the strategy profile $s$, Player $j^{t}$ accepts $x^{t}$ if $\left[x^{t}=\rho^{t}(h)\right]$ or $\left[x_{j}^{t} \geq \delta \bar{b}\right]$, and rejects otherwise. We consider three cases: 
1. $x^{t}=\rho^{t}(h)$,

2. $x^{t} \neq \rho^{t}(h)$ and $x_{j}^{t} \geq \delta \bar{b}$,

3. $x^{t} \neq \rho^{t}(h)$ and $x_{j}^{t}<\delta \bar{b}$.

The behavior of Player $j^{t}$ is irrelevant if Player $k^{t}$ responds after Player $j^{t}$ and accepts. So in the following we only need to consider the case where Player $k^{t}$ moves before Player $j^{t}$ or Player $k^{t}$ moves after Player $j^{t}$ and rejects.

Case 1. $x^{t}=\rho^{t}(h)$.

Note that if $x^{t}=\rho^{t}(h)$, then $x_{j}^{t} \geq \delta \underline{b}$. Indeed, if $x^{t}=a$,then $x_{j}^{t} \geq \delta \underline{b}$. Moreover, any proposal described by Tables 1 and 2 satisfies $x_{j}^{t}=\delta \underline{b}$ or $x_{j}^{t}=\delta \bar{b}$. According to the strategy profile $s$, Player $j^{t}$ accepts, which leads to a payoff of $\delta^{t} x_{j}^{t} \geq \delta^{t+1} \underline{b}$. If Player $j^{t}$ rejects $x^{t}$, this leads to an expected payoff of $\delta^{t+1}(1-\delta \bar{b}) / 3=\delta^{t+1} \underline{b}$. So rejection is not a profitable deviation.

Case 2. $x^{t} \neq \rho^{t}(h)$ and $x_{j}^{t} \geq \delta \bar{b}$.

According to the strategy profile $s$, Player $j^{t}$ accepts, which leads to a payoff of at least $\delta^{t+1} \bar{b}$. If Player $j^{t}$ deviates and rejects $x^{t}$, this leads to an expected payoff of $\delta^{t+1}((1-\delta \underline{b}) / 3+2 \delta \bar{b} / 3)=\delta^{t+1} \bar{b}$. So rejection is not a profitable deviation.

Case 3. $x^{t} \neq \rho^{t}(h)$ and $x_{j}^{t}<\delta \bar{b}$.

According to the strategy profile $s$, Player $j^{t}$ rejects, which leads to an expected payoff of $\delta^{t+1}((1-\delta \underline{b}) / 3+2 \delta \bar{b} / 3)=\delta^{t+1} \bar{b}$. If Player $j^{t}$ deviates and accepts, he receives a payoff less than or equal to $\delta^{t+1} \bar{b}$. So acceptance is not a profitable deviation.

We have checked that responders have no profitable one-shot deviations. We now turn our attention to the proposer. The following claim states that whenever a proposer deviates from $s$ and demands a share larger than $1-\delta \bar{b}$, the proposal will be rejected.

Claim A.4 Consider a history $h=\left(\pi^{0}, x^{0}, \ldots, \pi^{t}\right)$. A proposal $x^{t} \neq \rho^{t}(h)$ such that $x_{p^{t}}^{t}>$ $1-\delta \bar{b}$ is rejected by both responders.

Proof: Note that $x_{j}^{t}+x_{k}^{t}<\delta \bar{b}$. Since by definition Player $k^{t}$ is the responder who is offered the smaller share, we have $x_{k}^{t}<\delta \bar{b} / 2$. Moreover, the assumption $\delta \leq 3 / 5$ implies $\delta \bar{b} / 2 \leq \delta \underline{b}$, thus $x_{k}^{t}<\delta \underline{b}$. It follows by the definition of $s$ that Player $k^{t}$ rejects the proposal $x^{t}$. Player $j^{t}$ rejects the proposal $x^{t}$ since $x_{j}^{t}<\delta \bar{b}$.

The next claim states that a proposer does not have a profitable one-shot deviation. 
Claim A.5 Consider a history $h=\left(\pi^{0}, x^{0}, \ldots, \pi^{t}\right)$. Player $p^{t}$ has no profitable one-shot deviation from $s$ at $h$.

Proof: If $\rho^{t}(h)=a$, then $a_{p^{t}}^{t} \geq 1-\delta \bar{b}$. Moreover, any proposal $x$ described in both Tables 1 and 2 satisfies $x_{p^{t}}=1-\delta \bar{b}$ or $x_{p^{t}}=1-\delta \underline{b}$. Since the proposal $\rho^{t}(h)$ is accepted, it follows that following $s$ leads to a payoff greater than or equal to $\delta^{t}(1-\delta \bar{b})$ for Player $p^{t}$.

If Player $p^{t}$ proposes $x^{t} \neq \rho^{t}(h)$ such that $x_{p^{t}}^{t}>1-\delta \bar{b}$, then the proposal is rejected by Claim A.4 and the expected payoff for Player $p^{t}$ is equal to $\delta^{t+1}((1-\delta \bar{b}) / 3+\delta \underline{b} / 3) \leq$ $\delta^{t}(1-\delta \bar{b})$.

If Player $p^{t}$ proposes $x^{t} \neq \rho^{t}(h)$ such that $x_{p^{t}}^{t} \leq 1-\delta \bar{b}$, then the proposal is either accepted and leads to a payoff of $\delta^{t} x_{p^{t}}^{t} \leq \delta^{t}(1-\delta \bar{b})$, or rejected and leads to an expected payoff of $\delta^{t+1}((1-\delta \bar{b}) / 3+\delta \underline{b} / 3) \leq \delta^{t}(1-\delta \bar{b})$. In both cases, the payoff of Player $p^{t}$ is less than or equal to $\delta^{t}(1-\delta \bar{b})$.

\section{The proof of Theorem 3.1 for $\delta>3 / 5$}

Throughout this subsection it is assumed that $\delta>3 / 5$. The proof of the previous subsection breaks down, since Claim A.4 is no longer true. If $\delta>3 / 5$, it is no longer guaranteed that a proposal $x^{t} \neq \rho^{t}(h)$ such that $x_{p^{t}}^{t}>1-\delta \bar{b}$ is rejected by responder $k^{t}$, since it is not necessarily the case that $x_{k}^{t} \leq \delta \underline{b}$. We therefore change the construction of the strategy profile $s$ and replace Table 2 by Table 3 . As before Table 1 gives the values for $f\left(p^{t}, x^{t}, j^{t}, k^{t}, p^{t+1}, y^{t}\right)$ when $x^{t}=y^{t}$ and Table 3 when $x^{t} \neq y^{t}$. For $x_{k}^{t} \in[0,1 / 2], \theta\left(x_{k}^{t}\right)$ measures the excess of $x_{k}^{t}$ over $\delta \underline{b}$ when $\delta \underline{b}<x_{k}^{t}<\delta \bar{b} / 2$ and is equal to zero otherwise. Notice that when $\delta>3 / 5$, it holds that $\underline{b}<\bar{b} / 2$. More precisely, $\theta\left(x_{k}^{t}\right)$ is defined by

$$
\theta\left(x_{k}^{t}\right)= \begin{cases}x_{k}^{t} / \delta-\underline{b}, & \text { if } \delta \underline{b}<x_{k}^{t}<\delta \bar{b} / 2, \\ 0, & \text { otherwise. }\end{cases}
$$

If $\theta\left(x_{k}^{t}\right)=0$, then Table 3 is identical to Table 2 . In case $\theta\left(x_{k}^{t}\right) \neq 0$, Player $j^{t}$ obtains an expected payoff of $\bar{b}-\theta\left(x_{k}^{t}\right)$. Player $j^{t}$ is still rewarded for rejecting a proposal that was not described by the strategy profile, but he shares a part of the reward with Player $k^{t}$, who receives the remainder $x_{k}^{t} / \delta$. This makes Player $k^{t}$ indifferent between accepting and rejecting $x_{k}^{t}$.

We define the strategy profile $s$ as follows:

1. For each $t \geq 0$, for each history $h=\left(\pi^{0}, x^{0}, \ldots, \pi^{t}\right)$, Player $p^{t}$ proposes $\rho^{t}(h)$.

2. After history $h=\left(\pi^{0}, x^{0}, \ldots, \pi^{t}, x^{t}\right)$, Player $j^{t}$ accepts $x^{t}$ if 


\begin{tabular}{|c|c|c|c|c|c|}
\hline$p^{t}$ & $j^{t}$ & $k^{t}$ & $p^{t+1}=1$ & $p^{t+1}=2$ & $p^{t+1}=3$ \\
\hline 1 & 2 & 3 & $(1-\delta \bar{b}, \delta \bar{b}-\theta, \theta)$ & $(\delta \underline{b}, 1-\delta \underline{b}-\theta, \theta)$ & $(0, \delta \bar{b}-\theta, 1-\delta \bar{b}+\theta)$ \\
\hline 1 & 3 & 2 & $(1-\delta \bar{b}, \theta, \delta \bar{b}-\theta)$ & $(0,1-\delta \bar{b}+\theta, \delta \bar{b}-\theta)$ & $(\delta \underline{b}, \theta, 1-\delta \underline{b}-\theta)$ \\
\hline 2 & 1 & 3 & $(1-\delta \underline{b}-\theta, \delta \underline{b}, \theta)$ & $(\delta \bar{b}-\theta, 1-\delta \bar{b}, \theta)$ & $(\delta \bar{b}-\theta, 0,1-\delta \bar{b}+\theta)$ \\
\hline 2 & 3 & 1 & $(1-\delta \bar{b}+\theta, 0, \delta \bar{b}-\theta)$ & $(\theta, 1-\delta \bar{b}, \delta \bar{b}-\theta)$ & $(\theta, \delta \underline{b}, 1-\delta \underline{b}-\theta)$ \\
\hline 3 & 1 & 2 & $(1-\delta \underline{b}-\theta, \theta, \delta \underline{b})$ & $(\delta \bar{b}-\theta, 1-\delta \bar{b}+\theta, 0)$ & $(\delta \bar{b}-\theta, \theta, 1-\delta \bar{b})$ \\
\hline 3 & 2 & 1 & $(1-\delta \bar{b}+\theta, \delta \bar{b}-\theta, 0)$ & $(\theta, 1-\delta \underline{b}-\theta, \delta \underline{b})$ & $(\theta, \delta \bar{b}-\theta, 1-\delta \bar{b})$ \\
\hline
\end{tabular}

Table 3: $f\left(p^{t}, x^{t}, j^{t}, k^{t}, p^{t+1}, y^{t}\right)$, where $x^{t} \neq y^{t}$ and $\theta=\theta\left(x_{k}^{t}\right)$.

- $\left[x^{t}=\rho^{t}(h)\right]$ or

- $\left[x_{k}^{t} \leq \delta \underline{b}\right.$ and $\left.x_{j}^{t} \geq \delta \bar{b}\right]$ or

- $\left[\delta \underline{b}<x_{k}^{t}<\delta \bar{b} / 2\right.$ and $\left.x_{j}^{t} \geq \delta \bar{b}+\delta \underline{b}-x_{k}^{t}\right]$ or

- $\left[x_{k}^{t} \geq \delta \bar{b} / 2\right.$ and $\left.x_{j}^{t} \geq \delta \bar{b}\right]$,

and rejects otherwise.

3. After history $h=\left(\pi^{0}, x^{0}, \ldots, \pi^{t}, x^{t}\right)$, Player $k^{t}$ accepts $x^{t}$ if

- $\left[x^{t}=\rho^{t}(h)\right.$ and $\left.x_{k}^{t} \geq \delta^{2} \underline{b} / 3+\delta \underline{b}\right]$ or

- $\left[x^{t} \neq \rho^{t}(h)\right.$ and $\left.x_{k}^{t} \geq \delta \bar{b} / 2\right]$,

and rejects otherwise.

The proof of the fact that the strategy profile $s$ described above is an SPE is divided into six steps, five of which are the same as in the proof for $\delta \leq 3 / 5$. We need one additional step to provide a bound on $\theta\left(x_{k}^{t}\right)$.

Claim A.6 states that $s$ has the "no delay property."

Claim A.6 The strategy profile s has the following no delay property: if after a history $h=\left(\pi^{0}, x^{0}, \ldots, \pi^{t}\right)$ the players play according to the strategy profile $s$, then $\rho^{t}(h)$ is proposed and accepted by one of the responders and the game ends.

Proof: The claim is immediate from the definition of $s$. Indeed, the proposer proposes $x^{t}=\rho^{t}(h)$, which is accepted by Player $j^{t}$.

Claim A.7 shows that, after any responder history $h$, the responder with the smaller share, $k^{t}$, has no profitable one-shot deviations from $s$ at $h$. 
Claim A.7 Consider a history $h=\left(\pi^{0}, x^{0}, \ldots, \pi^{t}, x^{t}\right)$. Player $k^{t}$ has no profitable one-shot deviation from $s$ at $h$.

Proof: According to the strategy profile $s$, Player $k^{t}$ accepts $x^{t}$ if $\left[x^{t}=\rho^{t}(h)\right.$ and $x_{k}^{t} \geq$ $\left.\delta^{2} \underline{b} / 3+\delta \underline{b}\right]$ or $\left[x^{t} \neq \rho^{t}(h)\right.$ and $\left.x_{k}^{t} \geq \delta \bar{b} / 2\right]$, and rejects otherwise. We consider five cases:

1. $x^{t}=\rho^{t}(h)$ and $x_{k}^{t}<\delta^{2} \underline{b} / 3+\delta \underline{b}$ - the proof is the same as in Claim A.2,

2. $x^{t}=\rho^{t}(h)$ and $x_{k}^{t} \geq \delta^{2} \underline{b} / 3+\delta \underline{b}$ - the proof is the same as in Claim A.2,

3. $x^{t} \neq \rho^{t}(h)$ and $x_{k}^{t} \leq \delta \underline{b}$ - the proof is the same as in Claim A.2,

4. $x^{t} \neq \rho^{t}(h)$ and $\delta \underline{b}<x_{k}^{t}<\delta \bar{b} / 2$,

5. $x^{t} \neq \rho^{t}(h)$ and $x_{k}^{t} \geq \delta \bar{b} / 2$.

The behavior of Player $k^{t}$ is irrelevant if Player $j^{t}$ responds after Player $k^{t}$ and accepts. So in the following we only need to consider the case where Player $j^{t}$ moves before Player $k^{t}$ or Player $j^{t}$ moves after Player $k^{t}$ and rejects.

Case 4. $x^{t} \neq \rho^{t}(h)$ and $\delta \underline{b}<x_{k}^{t}<\delta \bar{b} / 2$.

According to the strategy profile $s$, Player $k^{t}$ rejects the proposal. Recalling that $\theta\left(x_{k}^{t}\right)=x_{k}^{t} / \delta-\underline{b}$ and using Equation (3.1), the resulting expected payoff is equal to $\delta^{t+1}\left(\frac{1}{3}\left(1-\delta \bar{b}+\theta\left(x_{k}^{t}\right)\right)+\frac{1}{3} \theta\left(x_{k}^{t}\right)+\frac{1}{3} \theta\left(x_{k}^{t}\right)\right)=\delta^{t+1}\left(\frac{1}{3}(1-\delta \bar{b})+\theta\left(x_{k}^{t}\right)\right)=\delta^{t+1}\left(\underline{b}+\theta\left(x_{k}^{t}\right)\right)=$ $\delta^{t} x_{k}^{t}$. If Player $k^{t}$ deviates and accepts, he obtains a payoff of $\delta^{t} x_{k}^{t}$. So acceptance is not a profitable deviation.

Case 5. $x^{t} \neq \rho^{t}(h)$ and $x_{k}^{t} \geq \delta \bar{b} / 2$.

According to the strategy profile $s$, Player $k^{t}$ accepts the proposal, which leads to a payoff of at least $\delta^{t} \delta \bar{b} / 2$. If Player $k^{t}$ deviates and rejects, he obtains an expected payoff of $\delta^{t+1}((1-\delta \bar{b}) / 3)=\delta^{t+1} \underline{b}<\delta^{t} \delta \bar{b} / 2$ for $\delta>3 / 5$. So rejection is not a profitable deviation.

Claim A.8 puts a bound on $\theta\left(x_{k}^{t}\right)$.

Claim A.8 For every $x_{k}^{t} \in[0,1 / 2]$, it holds that $\theta\left(x_{k}^{t}\right)<\delta(\bar{b}-\underline{b})$.

Proof: It follows from the definition that $\theta\left(x_{k}^{t}\right)<\frac{1}{2} \bar{b}-\underline{b}$. Hence

$$
\delta \bar{b}-\theta\left(x_{k}^{t}\right)>\delta \bar{b}-\frac{1}{2} \bar{b}+\underline{b}=\left(\delta-\frac{1}{2}\right) \bar{b}+\underline{b}>\left(\delta-\frac{1}{2}\right) 2 \underline{b}+\underline{b}=2 \delta \underline{b}>\delta \underline{b},
$$

where we have used the fact that $\delta>3 / 5$ and $\bar{b}>2 \underline{b}>0$. 
Claim A.9 shows that, after any responder history $h$, the responder with the higher share, $j^{t}$, has no profitable one-shot deviation from $s$ at $h$.

Claim A.9 Consider a history $h=\left(\pi^{0}, x^{0}, \ldots, \pi^{t}, x^{t}\right)$. Player $j^{t}$ has no profitable one-shot deviation from $s$ at $h$.

Proof: According to the strategy profile $s$, Player $j^{t}$ accepts $x^{t}$ if

- $\left[x^{t}=\rho^{t}(h)\right]$ or

- $\left[x_{k}^{t} \leq \delta \underline{b}\right.$ and $\left.x_{j}^{t} \geq \delta \bar{b}\right]$ or

- $\left[\delta \underline{b}<x_{k}^{t}<\delta \bar{b} / 2\right.$ and $\left.x_{j}^{t} \geq \delta \bar{b}+\delta \underline{b}-x_{k}^{t}\right]$ or

- $\left[x_{k}^{t} \geq \delta \bar{b} / 2\right.$ and $\left.x_{j}^{t} \geq \delta \bar{b}\right]$,

and rejects otherwise. We consider five cases:

1. $x^{t}=\rho^{t}(h)$,

2. $x^{t} \neq \rho^{t}(h)$ and $\left[x_{k}^{t} \leq \delta \underline{b}\right.$ or $\left.x_{k}^{t} \geq \delta \bar{b} / 2\right]$ and $x_{j}^{t} \geq \delta \bar{b}$ - the proof is the same as in Claim A.3,

3. $x^{t} \neq \rho^{t}(h)$ and $\left[x_{k}^{t} \leq \delta \underline{b}\right.$ or $\left.x_{k}^{t} \geq \delta \bar{b} / 2\right]$ and $x_{j}^{t}<\delta \bar{b}$,

4. $x^{t} \neq \rho^{t}(h)$ and $\delta \underline{b}<x_{k}^{t}<\delta \bar{b} / 2$ and $x_{j}^{t} \geq \delta \bar{b}+\delta \underline{b}-x_{k}^{t}$,

5. $x^{t} \neq \rho^{t}(h)$ and $\delta \underline{b}<x_{k}^{t}<\delta \bar{b} / 2$ and $x_{j}^{t}<\delta \bar{b}+\delta \underline{b}-x_{k}^{t}$.

The behavior of Player $j^{t}$ is irrelevant if Player $k^{t}$ responds after Player $j^{t}$ and accepts. So in the following we only need to consider the case where Player $k^{t}$ moves before Player $j^{t}$ or Player $k^{t}$ moves after Player $j^{t}$ and rejects.

Case 1. $x^{t}=\rho^{t}(h)$.

First we argue that if $x^{t}=\rho^{t}(h)$, then $x_{j}^{t} \geq \delta \underline{b}$. If $x^{t}=a$, then $x_{j}^{t} \geq \delta \underline{b}$. Moreover, any proposal in Table 1 satisfies $x_{j}^{t}=\delta \underline{b}$ or $x_{j}^{t}=\delta \bar{b}$. And any proposal in Table 3 offers the responders the shares $\delta \underline{b}$ and $\theta\left(x_{k}^{t-1}\right)$, or the shares $\delta \bar{b}-\theta\left(x_{k}^{t-1}\right)$ and 0 , or $\delta \bar{b}-\theta\left(x_{k}^{t-1}\right)$ and $\theta\left(x_{k}^{t-1}\right)$. In view of Claim A.8, in either case one of the two responders is offered at least $\delta \underline{b}$, so $x_{j}^{t} \geq \delta \underline{b}$.

According to the strategy profile $s$, Player $j^{t}$ accepts, leading to a payoff $\delta^{t} x_{j}^{t} \geq \delta^{t+1} \underline{b}$. If Player $j^{t}$ deviates and rejects $x^{t}$, this leads to an expected payoff of $\delta^{t+1}(1-\delta \bar{b}) / 3=\delta^{t+1} \underline{b}$. So rejection is not a profitable deviation. 
Case 3. $x^{t} \neq \rho^{t}(h)$ and $\left[x_{k}^{t} \leq \delta \underline{b}\right.$ or $\left.x_{k}^{t} \geq \delta \bar{b} / 2\right]$ and $x_{j}^{t}<\delta \bar{b}$.

According to the strategy profile $s$, Player $j^{t}$ rejects, leading to an expected payoff of $\delta^{t+1}((1-\delta \underline{b}) / 3+2 \delta \bar{b} / 3)=\delta^{t+1} \bar{b}$. If Player $j^{t}$ deviates and accepts, he receives a payoff less than $\delta^{t+1} \bar{b}$. So acceptance is not a profitable deviation.

Case 4. $x^{t} \neq \rho^{t}(h)$ and $\delta \underline{b}<x_{k}^{t}<\delta \bar{b} / 2$ and $x_{j}^{t} \geq \delta \bar{b}+\delta \underline{b}-x_{k}^{t}$.

According to the strategy profile $s$, Player $j^{t}$ accepts, leading to a payoff of at least $\delta^{t+1} \bar{b}+\delta^{t+1} \underline{b}-\delta^{t} x_{k}^{t}$. If Player $j^{t}$ deviates and rejects, this leads to an expected payoff of

$$
\delta^{t+1}\left(\frac{1}{3}\left(1-\delta \underline{b}-\theta\left(x_{k}^{t-1}\right)\right)+\frac{2}{3}\left(\delta \bar{b}-\theta\left(x_{k}^{t-1}\right)\right)\right)=\delta^{t+1}\left(\bar{b}-\theta\left(x_{k}^{t-1}\right)\right)=\delta^{t+1} \bar{b}+\delta^{t+1} \underline{b}-\delta^{t} x_{k}^{t},
$$

where we used (3.1) and the fact that $\theta\left(x_{k}^{t}\right)=x_{k}^{t} / \delta-\underline{b}$. So rejection is not a profitable deviation.

Case 5. $x^{t} \neq \rho^{t}(h)$ and $\delta \underline{b}<x_{k}^{t}<\delta \bar{b} / 2$ and $x_{j}^{t}<\delta \bar{b}+\delta \underline{b}-x_{k}^{t}$.

According to the strategy profile $s$, Player $j^{t}$ rejects, leading to an expected payoff of $\delta^{t+1} \bar{b}+\delta^{t+1} \underline{b}-\delta^{t} x_{k}^{t}$. If Player $j^{t}$ deviates and accepts, this gives him a payoff of at most $\delta^{t+1} \bar{b}+\delta^{t+1} \underline{b}-\delta^{t} x_{k}^{t}$. So acceptance is not a profitable deviation.

Claim A.10 shows that a proposal $x^{t} \neq \rho^{t}(h)$ which gives to the proposer more than $1-\delta \bar{b}$ is rejected by both responders.

Claim A.10 Consider a history $h=\left(\pi^{0}, x^{0}, \ldots, \pi^{t}\right)$. A proposal $x^{t} \neq \rho^{t}(h)$ such that $x_{p^{t}}^{t}>1-\delta \bar{b}$ is rejected by both responders.

Proof: Note that $x_{j}^{t}+x_{k}^{t}<\delta \bar{b}$. Since $k^{t}$ is the responder who is offered the smaller share, we have $x_{k}^{t}<\delta \bar{b} / 2$. We consider 2 cases:

1. $x_{k}^{t} \leq \delta \underline{b}$,

2. $\delta \underline{b}<x_{k}^{t}<\delta \bar{b} / 2$.

Case 1. $x_{k}^{t} \leq \underline{\delta} \underline{b}$.

According to the strategy profile $s$, Player $k^{t}$ rejects the proposal as $x_{k}^{t} \leq \delta \underline{b}<\delta \bar{b} / 2$.

According to the strategy profile $s$, Player $j^{t}$ rejects the proposal as $x_{j}^{t}<\delta \bar{b}$.

Case 2. $\delta \underline{b}<x_{k}^{t}<\delta \bar{b} / 2$.

According to the strategy profile $s$, Player $k^{t}$ rejects the proposal as $x_{k}^{t}<\delta \bar{b} / 2$.

According to the strategy profile $s$, Player $j^{t}$ rejects the proposal as

$$
x_{j}^{t} \leq 1-x_{p^{t}}^{t}-x_{k}^{t}<1-(1-\delta \bar{b})-x_{k}^{t}=\delta \bar{b}-x_{k}^{t}<\delta \bar{b}+\delta \underline{b}-x_{k}^{t} .
$$


Claim A.11 shows that after any proposer history $h$, the proposer has no profitable one-shot deviation from $s$ at $h$.

Claim A.11 Consider a history $h=\left(\pi^{0}, x^{0}, \ldots, \pi^{t-1}, x^{t-1}\right)$. Player $p^{t}$ has no profitable one-shot deviation from $s$ at $h$.

Proof: First we argue that following $s$ leads to a payoff of at least $\delta^{t}(1-\delta \bar{b})$ for Player $p^{t}$. If $\rho^{t}(h)=a$, then $a_{p^{t}}^{t} \geq 1-\delta \bar{b}$. Otherwise, according to $s$, the proposer demands one of the four shares: $1-\delta \bar{b}, 1-\delta \underline{b}, 1-\delta \bar{b}+\theta\left(x_{k}^{t}\right)$, and $1-\delta \underline{b}-\theta\left(x_{k}^{t}\right)$. Each of these is at least $1-\delta \bar{b}$, where the fact that $1-\delta \underline{b}-\theta\left(x_{k}^{t}\right)$ is at least $1-\delta \bar{b}$ follows from Claim A.8.

If Player $p^{t}$ proposes $x^{t} \neq \rho^{t}(h)$ such that $x_{p^{t}}^{t}>1-\delta \bar{b}$, then the proposal is rejected according to Claim A.10 and the expected payoff for Player $p^{t}$ is equal to $\delta^{t+1}((1-\delta \bar{b}) / 3+$ $\delta \underline{b} / 3) \leq \delta^{t}(1-\delta \bar{b})$

If Player $p^{t}$ proposes $x^{t} \neq \rho^{t}(h)$ such that $x_{p^{t}}^{t} \leq 1-\delta \bar{b}$, then the proposal is either accepted and leads to a payoff of $\delta^{t} x_{p^{t}}^{t} \leq \delta^{t}(1-\delta \bar{b})$, or is rejected and leads to an expected payoff of $\delta^{t+1}((1-\delta \bar{b}) / 3+\delta \underline{b} / 3) \leq \delta^{t}(1-\delta \bar{b})$.

\section{References}

Banks, J., And J. Duggan (2000), "A Bargaining Model of Collective Choice," American Political Science Review, 94, 73-88.

Baron, D.P., And J.A. Ferejohn (1989), "Bargaining in Legislatures," American Political Science Review, 83, 1181-1206.

Baron, D., And E. Kalai (1993), "The Simplest Equilibrium of a Majority-Rule Division Game," Journal of Economic Theory, 61, 290-301.

Breitmoser, Y., And J.H.W. TAN (2010), "Generosity in Bargaining: Fair or Fear?," MPRA Paper No. 27444, pp. 1-35.

Chatteruee, K., B. Dutta, D. Ray, and K. Sengupta (1993), "A Noncooperative Theory of Coalitional Bargaining," Review of Economic Studies, 60, 463-477.

Cho, S.J., And J. Duggan (2009), "Bargaining Foundations of the Median Voter Theorem," Journal of Economic Theory, 144, 851-868.

Cho, S.J., And J. Duggan (2013), "A Folk Theorem for the One-dimensional Spatial Bargaining Model," Working Paper, 1-16.

Diermeier, D., AND R. Morton (2006), "Experiments in Majoritarian Bargaining," in D. AustenSmith and J. Duggan (eds.), Social Choice and Strategic Decisions: Essays in Honor of Jeffrey S. Banks, Springer, Heidelberg, pp. 201-226. 
Eraslan, H. (2002), "Uniqueness of Stationary Equilibrium Payoffs in the Baron-Ferejohn Model," Journal of Economic Theory, 103, 11-30.

Eraslan, H., And A. McLennan (2013), "Uniqueness of Stationary Equilibrium Payoffs in Coalitional Bargaining," Journal of Economic Theory, 148, 2195-2222.

Fréchette, G., J.H. Kagel, And S.F. Lehrer (2003), "Bargaining in Legislatures: An Experimental Investigation of Open versus Closed Amendment Rules," American Political Science Review, 97, $221-232$.

Fréchette, G., J.H. Kagel, And M. Morelli (2005), "Nominal Bargaining Power, Selection Protocol, and Discounting in Legislative Bargaining," Journal of Public Economics, 89, 1497-1517.

Fudenberg, D., And J. Tirole (1991), Game Theory, MiT Press, Cambridge, Massachusetts.

Harrington, J.E. JR. (1990), "The Power of the Proposal Maker in a Model of Endogenous Agenda Formation," Public Choice, 64, 1-20.

Kagel, J.H, H. Sung, And E. Winter (2010), "Veto Power in Committees: An Experimental Study," Experimental Economics, 13, 167-188.

Maskin, E.S., AND J. Tirole (2001), "Markov Perfect Equilibrium, I. Observable Actions," Journal of Economic Theory, 100, 191-219.

McKelvey, R.D. (1991), "An Experimental Test of a Stochastic Game Model of Committee Bargaining," in T. Palfrey (ed.), Laboratory Research in Political Economy, University of Michigan Press, Ann Arbor, pp. 139-69.

Miller, L., And C. Vanberg (2013), "Decision Costs in Legislative Bargaining: An Experimental Analysis," Public Choice, 155, 373-394.

Norman, P. (2002), "Legislative Bargaining and Coalition Formation," Journal of Economic Theory, 102, 322-353.

Rubinstein, A. (1982), "Perfect Equilibrium in a Bargaining Model," Econometrica, 50, 97-109.

Rubinstein, A. (1991), "Comments on the Interpretation of Game Theory," Econometrica, 59, 909-924. 